\title{
Comparative transcriptome analysis of panicle development under heat stress in two rice (Oryza sativa L.) cultivars differing in heat tolerance
}

\author{
Yaliang Wang ${ }^{\text {Equal first author, } 1}$, Yikai Zhang ${ }^{\text {Equal first author, } 1}{ }^{1}$, Qiang Zhang ${ }^{1}$, Yongtao Cui ${ }^{1}$, Jing Xiang ${ }^{1}$, Huizhe Chen ${ }^{1}$, \\ Guohui Hu ${ }^{1}$, Yanhua Chen ${ }^{1}$, Xiaodan Wang ${ }^{1}$, Defeng Zhu ${ }^{\text {Corresp., } 1}$, Yuping Zhang ${ }^{\text {Corresp. } 1}$ \\ ${ }^{1}$ State Key Laboratory of Rice Biology, China National Rice Research Inistitute, Hangzhou, Zhejiang, China \\ Corresponding Authors: Defeng Zhu, Yuping Zhang \\ Email address: cnrice@qq.com, cnrrizyp@163.com
}

Heat stress inhibits rice panicle development and reduces the spikelet number per panicle. This study investigated the mechanism involved in heat-induced damage to panicle development and spikelet formation in rice cultivars that differ in heat tolerance. Transcriptome data from developing panicles grown at $40^{\circ} \mathrm{C}$ or $32^{\circ} \mathrm{C}$ were compared for two rice cultivars: heat-tolerant Huanghuazhan and heat-susceptible IR36. Of the differentially expressed genes (DEGs), 4,070 heat stress-responsive genes were identified, including 1,688 heat-resistant-cultivar-related genes (RHR), 707 heat-susceptible-cultivarrelated genes (SHR), and 1,675 common heat stress-responsive genes. A Gene ontology (GO) analysis showed that the DEGs in the RHR category were significantly enriched in 54 gene ontology terms, some of which improved heat tolerance, including those in the WRKY, HD-ZIP, ERF, and MADS transcription factor families. A Kyoto Encyclopedia of Genes and Genomes (KEGG) analysis showed that the DEGs in the RHR and SHR categories were enriched in 15 and 11 significant metabolic pathways, respectively. Improved signal transduction capabilities of endogenous hormones under high temperature seemed to promote heat tolerance, while impaired starch and sucrose metabolism under high temperature might have inhibited young panicle development. Our transcriptome analysis provides insights into the different molecular mechanisms of heat stress tolerance in developing rice. 


\section{Comparative transcriptome analysis of panicle}

\section{2 development under heat stress in two rice (Oryza sativa}

\section{L.) cultivars differing in heat tolerance}

4 Yaliang Wang", Yikai Zhang\#, Qiang Zhang, Yongtao Cui, Jing Xiang, Huizhe Chen, Guohui Hu,

5 Yanhua Chen, Xiaodan Wang, Defeng Zhu*, Yuping Zhang*

7 State Key Laboratory of Rice Biology, China National Rice Research Institute, Hangzhou, 8 Zhejiang, China

\# The first two authors contributed equally to this paper

*Corresponding authors:

\section{Yuping Zhang, E-mail: cnrrizyp@,163.com}

\section{Abstract}

Heat stress inhibits rice panicle development and reduces the spikelet number per panicle. This study investigated the mechanism involved in heat-induced damage to panicle development and spikelet formation in rice cultivars that differ in heat tolerance. Transcriptome data from developing panicles grown at $40^{\circ} \mathrm{C}$ or $32^{\circ} \mathrm{C}$ were compared for two rice cultivars: heat-tolerant Huanghuazhan and heat-susceptible IR36. Of the differentially expressed genes (DEGs), 4,070 heat stress-responsive genes were identified, including 1,688 heat-resistant-cultivar-related genes (RHR), 707 heat-susceptible-cultivar-related genes (SHR), and 1,675 common heat stressresponsive genes. A Gene ontology (GO) analysis showed that the DEGs in the RHR category were significantly enriched in 54 gene ontology terms, some of which improved heat tolerance, including those in the WRKY, HD-ZIP, ERF, and MADS transcription factor families. A Kyoto Encyclopedia of Genes and Genomes (KEGG) analysis showed that the DEGs in the RHR and SHR categories were enriched in 15 and 11 significant metabolic pathways, respectively. Improved signal transduction capabilities of endogenous hormones under high temperature seemed to promote heat tolerance, while impaired starch and sucrose metabolism under high temperature might have inhibited young panicle development. Our transcriptome analysis provides insights into the different molecular mechanisms of heat stress tolerance in developing rice. 
34

Key words: Rice; Panicle development; Heat stress; Transcriptome analysis

\section{Introduction}

Climate change is predicted to increase the average global temperatures by $0.3-4.8^{\circ} \mathrm{C}$ by the end of the 21st century (Stocher et al. 2013). Unusually high temperatures occur frequently during the rice growing season (Dwivedi et al. 2015; Tao et al. 2013), and cause reductions in the yield and quality in several rice producing regions, including China, India, and Japan (Anand et al. 2018; Morita et al. 2016; Wang et al. 2019). The primary cause of rice yield reductions is a reduction in spikelet fertility due to high temperatures during the flowering period (Espe et al. 2017). Rice quality is also influenced by high temperature, which causes carbohydrate metabolism disorders (Yamakawa \& Hakata 2010). As climate change has intensified, extremely high temperatures above $40^{\circ} \mathrm{C}$ have become more frequent. Such high temperatures inhibit rice panicle development, reduce the spikelet number by 5\%-15\%, and aggravate rice yield losses (Wang et al. 2017).

High temperatures adversely affect floral development by reducing the antioxidant capacity, inhibiting nutrient accumulation, and degenerating tapetal cells (Prasad et al. 2017). A previous study showed that high temperature $\left(39^{\circ} \mathrm{C}\right)$ conditions downregulated certain genes related to tapetum function, pollen adhesion, and germination, including OsINV4 and OSMST8, which influenced spikelet fertilization (Endo et al. 2009). In addition, sugar and endogenous hormone metabolism under high temperature reportedly plays an important role in pollen formation in both rice and cotton (Islam et al. 2018; Min et al. 2014). At the rice ripening stage, high temperature induces early termination of grain filling (Kim et al. 2011). Grain chalkiness increases under a mean temperature greater than $32^{\circ} \mathrm{C}$, resulting in the deterioration of eating and cooking quality, which are both closely linked to starch and sucrose metabolism (Zhong et al. 2010). Transcriptome analysis has shown that high temperatures influence the expression of genes involved in the inhibition of sucrose degradation and starch biosynthesis while promoting starch degradation and storage proteins synthesis (Yamakawa \& Hakata 2010; Yamakawa et al. 2007). Takehara et al. (2018) reported that the upregulation of OSSUS3, which encodes sucrose synthase, improved hightemperature tolerance.

The panicle initiation stage is an important period of spikelet proliferation. Dry matter accumulation is essential for panicle development; however, the pathway for carbohydrate accumulation during spikelet formation under heat stress remains vague. The reduction in spikelet number that occurs under high temperature conditions has been associated with heat-induced phytohormone changes, especially enhanced cytokinin degradation (Wu et al. 2017; Wu et al. 
2016). The number of spikelets per panicle is determined by spikelet differentiation and degeneration. Spikelet differentiation is correlated with dry matter accumulation and influenced by environmental factors (Liu et al. 2005). Ding et al. (2016) reported that hormone metabolism, the stress response, carbohydrate metabolism and transport, and protein degradation were regulated to influence panicle initiation. Additionally, certain genes, such as MADS-box genes, are related to panicle initiation (Kang et al. 2013; Kobayashi et al. 2012). Quantitative trait loci for spikelet degeneration have been identified (Yamagishi et al. 2004), and the genes SP1, ASP1, TUT1, PAA2, and OsALMT7 have been found to control spikelet degeneration (Bai et al. 2015; Heng et al. 2018; Li et al. 2010). However, the mechanism of panicle development under high temperature conditions is still unclear. In this study, an RNA-Seq analysis was used to explore the mechanism of heat tolerance during panicle development. Huanghuazhan (HHZ) is a heat-tolerant rice cultivar widely grown in the middle and lower reaches of the Yangtze River in China (Cao et al. 2009; Zhou et al. 2012). IR36 is a heat-susceptible cultivar (Fang et al. 2006) and a parental line of HHZ. In the current study, we investigated transcriptome differences between these two cultivars exposed to different temperatures $40^{\circ} \mathrm{C}$ and $32^{\circ} \mathrm{C}$, during the spikelet differentiation stage. We identified differentially expressed genes (DEGs) in young panicles of the two cultivars under the two temperature treatments and performed Gene Ontology (GO) enrichment and the Kyoto Encyclopedia of Genes and Genomes (KEGG) analysis. This work improves our understanding of the molecular mechanism underlying the heat-induced inhibition of spikelet development and provides important insights into rice breeding.

\section{Materials and methods}

\section{Plant materials and heat stress treatments}

We used the rice cultivars HHZ and IR36 in this study. Pre-germinated seeds were sown in seed trays filled with a mixture of vermiculite (20\%), charcoal (30\%), soil (40\%), and slow-release fertilizer $(10 \%)$. After 20 days, the seedlings were transplanted into pots with four seedlings per pot. Each pot $(24 \mathrm{~cm}$ length $\times 22.5 \mathrm{~cm}$ width $\times 21.5 \mathrm{~cm}$ height $)$ contained $10 \mathrm{~kg}$ air-dried paddy soil. Pots were kept under natural environmental conditions (the average temperature was 30$\left.35^{\circ} \mathrm{C}\right)$.

Before seedlings were transplanted into pots, fertilizer was applied to each pot based on a field application rate of $14 \mathrm{~kg}$ nitrogen per $666.7 \mathrm{~m}^{2}$. Before transplanting into the pots, $3.5 \mathrm{~g}$ compound fertilizer (nitrogen: phosphorus: potassium $=15 \%: 15 \%: 15 \%$ ) was applied to each pot. At the tillering stage, $0.6 \mathrm{~g}$ urea was supplemented in each pot. At panicle initiation, $0.6 \mathrm{~g}$ urea and $0.5 \mathrm{~g}$ potassium chloride were also applied to each pot. Pests, diseases, and weeds were intensively controlled. 
Automatic growth chambers (Qiushi Environment Corporation, Hangzhou, China) were used to conduct the temperature treatments. Plants were moved to the growth chambers on the approximate date of spikelet differentiation when the panicle length was approximately $0.2 \mathrm{~cm}$ (around 60-70 $\mathrm{d}$ after seed sowing). The high-temperature $\left(40^{\circ} \mathrm{C}\right)$ and control temperature $\left(32^{\circ} \mathrm{C}\right)$ treatments were implemented for eight hours each day from 9:30 to 17:30 h (the setting details are shown in Table S1) for nine days. The humidity in the chambers was maintained at $75-80 \%$. Rice plants were grown under natural ambient conditions during all growth stages before and after the temperature treatments. Each treatment contained three replicates with 20 pots per replicate.

\section{Panicle and spikelet morphology}

Ten main tillers were sampled per replicate on day 9 of treatment at $40^{\circ} \mathrm{C}$ or $32^{\circ} \mathrm{C}$ to investigate the development of young panicles at high temperature.

Spikelet differentiation or degeneration of the main tiller panicles was determined at the heading stage. The number of degenerated spikelets was calculated by counting the vestiges present on the panicles. The number of differentiated spikelets was the sum of the surviving and degenerated spikelets. The proportion of degenerated spikelets was then calculated.

Spikelet morphology was observed under a stereomicroscope (Olympus SZX7, Olympus Corporation, Tokyo, Japan) and the glume length and width ( $\mathrm{mm}$ )were measured at $0.63 \mathrm{x}$ and $2.5 \mathrm{x}$ using the microscale in the Image Pro-Plus 5.1 image processing software (Olympus SZX7, Olympus Corporation, Tokyo, Japan). Fifteen spikelets were collected from the upper, middle and lower parts of each panicle, with five panicles sampled for each replicate.

\section{RNA extraction, transcriptome sequencing, and mapping}

After nine days at the $40{ }^{\circ} \mathrm{C}$ or $32{ }^{\circ} \mathrm{C}$ treatment, young panicles from 20 main tillers were collected for each replicate at 12:00-13:00 and immediately frozen in liquid nitrogen. In quick succession, TRIzol reagent (Invitrogen, Carlsbad, CA, USA) was used to extract total RNA from the young panicles according to the manufacturer's instructions. A TruSeq RNA Sample Preparation Kit (Illumina Inc., CA, USA) was used to generate 12 sequencing libraries according to the manufacturer's instructions. The sequencing libraries were then sequenced on a HiSeq platform (Illumina, Inc., CA, USA). High-quality sequence reads were obtained by filtering the raw data and then compared to the 9311-reference genome (Oryza_indica.ASM465v1.dna.toplevel. fa) obtained from http://www.ensembl.org/. The raw RNA sequence data were submitted to the NCBI Sequence Read Archive with accession number PRJNA508820.

\section{Gene expression level and differential expression analysis}


We used HTSeq (0.9.1) to statistically compare the read count values of each gene, which represent the original expression of each gene. Fragments per kilobase of transcript per million mapped reads (FPKM) was used to standardize the expression. Next, we used DESeq (1.30.0) to analyze the differential expression of genes with the following screening conditions: an expression difference of $\mid \log 2$ foldChange $\mid>1$ and a significant $P$-value $<0.05$.

\section{GO and KEGG enrichment analysis of DEGs}

For the GO enrichment analysis of DEGs, we used the Singular Enrichment Analysis tool in AgriGO (http://bioinfo.cau.edu.cn/agriGO/analysis.php) with the default parameters, and a $P$ value $\leqslant 0.05$ indicated significant enrichment. The KEGG enrichment analysis of DEGs was performed using KOBAS software with default parameters and a $P$-value $\leqslant 0.05$ indicated significant pathway enrichment.

\section{Verification of RNA-Seq by quantitative real-time PCR (qRT-PCR)}

First-strand cDNA was synthesized using ReverTra Ace qPCR RT Master Mix with gDNA Remover (Toyobo, Osaka, Japan) according to the manufacturer's instructions. The qRT-PCR analyses were performed using an Applied Biosystems 7500 Real-Time PCR system with Power SYBR Green PCR Master Mix (Applied Biosystems, Carlsbad, CA, USA). The primers used for qRT-PCR are listed in Supplementary Table S2. The $O s U B Q$ gene was used as an internal control. Relative gene expression levels were determined from the equation $2^{-\Delta \Delta C T}$ (Czechowski et al. 2004), where $\Delta \Delta \mathrm{CT}$ represents $\Delta \mathrm{CT}$ (target gene of interest) $\Delta \mathrm{CT}$ (control gene).

\section{Statistical analyses}

Microsoft Excel 2016 (Microsoft Inc., Redmond, WA, USA) was employed for data collection. The panicle and spikelet morphological data collected for the $40^{\circ} \mathrm{C}$ and $32^{\circ} \mathrm{C}$ treatments (mean of three replicates) were statistically analyzed by Student's t-test $(P<0.05)$. Graphs were created using Origin 9.1 (Ver. 9.1, OriginLab, Northampton, MA, USA).

\section{Results}

\section{Spikelet development at high temperature}

A preliminary experiment showed a significant difference in panicle development, which was measured as spike differentiation, after nine days of the high-temperature treatment. The results reported in the current study are consistent with these preliminary findings. Spikelet differentiation inhibited young panicle growth after nine days of the high temperature treatment (Fig. 1). After 
174 the temperature treatments, the panicles required an additional 15-20 days to complete growth.

175 Compared to the control temperature treatment, the high temperature treatment reduced

176

177

178

179

180

181

182

183

184

185

186

187

188

189

190

191

192

193

194

195

196

197

198

199

200

201

202

203

204

205

206

207

208

209 spikelet survival by $22.3 \%(P<0.05)$ for HHZ and 53.6\% $(P<0.05)$ for IR36. With high temperature, the number of differentiated spikelets decreased by $9.6 \%$ and $33.2 \%(P<0.05)$ for HHZ and IR36, respectively, and the proportion of degenerated spikelets significantly increased by $32.3 \%(P<0.05)$ and $67.4 \%(P<0.05)$, respectively. In addition, the heat treatment reduced the glume length by $10.3 \%(P<0.05)$ for HHZ and by $16.0 \%(P<0.05)$ for IR36 and reduced the glume width by $12.0 \%(P<0.0 .5)$ and $8.0 \%(P<0.05)$, respectively. The reductions in spikelet number and size led to reductions in panicle weight of $33.2 \%(P<0.05)$ for $\mathrm{HHZ}$ and $67.7 \%(P<$ 0.05 ) for IR36. The larger reduction in panicle weight in IR36 suggests that high temperature has a greater effect on young panicle development in heat susceptible cultivars (Table 1).

\section{Transcriptome analysis}

Under the $32^{\circ} \mathrm{C}$ control temperature, a total of 44.2 million and 48.9 million raw reads were obtained from HHZ (referred to as HHZ_32) and IR36 (referred to as IR36_32), respectively. Under the $40^{\circ} \mathrm{C}$ treatment, a total of 45.5 million raw reads were obtained from both $\mathrm{HHZ}$ (HHZ_40) and IR36 (IR36_40) (Table 2 and Table S3). More than 99.0\% clean reads were obtained for the downstream analysis. The results of RNA sequence mapping indicated that 85.8$88.0 \%$ of the clean reads could be mapped onto the reference genome and most were uniquely mapped (Table 2).

\section{Identification of DEGs}

To compare the differences between the two cultivars at $40^{\circ} \mathrm{C}$ and $32^{\circ} \mathrm{C}$, we used four comparison groups: HHZ_32 vs HHZ_40, IR36_32 vs IR36_40, IR36_40 vs HHZ_40, and IR36_32 vs HHZ_32. DEGs for the four groups were restricted to those with a $\mid \log 2$ fold change $>1$ and a $P$-value $<0.05$. With these criteria, 3,342, 2,469, 2,949, and 2,461 DEGs were detected for HHZ_32 vs HHZ_40, IR36_32 vs IR36_40, IR36_40 vs HHZ_40, and IR36_32 vs HHZ_32, respectively (Fig. 2). Significantly different gene expression was observed both between cultivars and between treatments. For HHZ, 1,794 genes were upregulated and 1,548 genes were downregulated in the $40^{\circ} \mathrm{C}$ treatment compared with the $32^{\circ} \mathrm{C}$ treatment (Fig. 2). Furthermore, 1,140 genes were upregulated and 1,329 genes were downregulated in IR36 under the $40^{\circ} \mathrm{C}$ treatment compared with the $32{ }^{\circ} \mathrm{C}$ treatment (Fig. 2). For comparisons within treatments, 1,408 genes were upregulated and 1,541 were downregulated in the IR36_40 vs HHZ_40 group and 893 genes were upregulated and 1,751 genes were downregulated in the IR36_32 vs HHZ_32 group (Fig.2c and 2d). 
210 Classification of DEGs

211 In all four groups, a total of 5,533 unique DEGs were identified, and they could be divided 212 into 15 disjointed subgroups (Fig. 3). Among the 15 subgroups, eight from the IR36_32 vs 213 HHZ_32 group were excluded from the analysis because they were not influenced by high 214 temperature. In addition, 1,157, 603, 524, and 402 DEGs were uniquely identified in the HHZ_32 215 vs HHZ_40, IR36_32 vs IR36_40, IR36_40 vs HHZ_40, and IR36_32 vs HHZ_32 groups, 216 respectively. The DEGs in groups that were responsive to high temperature could be further sorted 217 into three categories: heat-tolerance-cultivar-related genes (RHR, 1,688 genes), heat-susceptible218 cultivar-related genes (SHR, 707 genes), and common heat stress-response genes (CHR, 1,675 219 genes) (Table 3 and Table S4). The DEGs in the RHR category might have played an important role in heat tolerance, whereas the DEGs in the SHR category might be associated with heat injuries in the heat-susceptible cultivar.

\section{Analysis of GO annotation}

The purpose of the GO enrichment analysis was to obtain GO functional terms with significant enrichment of DEGs and thus reveal the possible functions of the DEGs. Of all DEGs, 2,307 (69.0\%), 1,680 (68.0\%), 1,832 (62.1\%), and 1,472 (59.8\%) DEGs were enriched in GO terms in HHZ_32 vs HHZ_40, IR36_32 vs IR36_40, IR36_40 vs HHZ_40, and IR36_32 vs HHZ_32 groups, respectively. There were 75, 11, 13, and 31 significant GO terms observed in HHZ_32 vs HHZ_40, IR36_32 vs IR36_40, IR36_40 vs HHZ_40, and IR36_32 vs HHZ_32, respectively (Fig. 4). The maximum number of DEGs was observed for the heterocycle biosynthetic process in the IR36_40 vs HHZ_40 group. In IR36_32 vs IR36_40 and HHZ_32 vs HHZ_40, the DEGs were enriched in the terms response to stimulus, response to temperature stimulus, and response to heat in the biological process category. Within the cellular component category, the DEGs were commonly enriched in the terms chromatin, DNA packaging complex, and nucleosome in the IR36_32 vs IR36_40 and HHZ_32 vs HHZ_40 groups. However, there were no common GO terms in the category of molecular function in the IR36_32 vs IR36_40 and HHZ_32 vs HHZ_40 groups.

We further identified GO term categories for DEGs in the RHR, SHR, and CHR categories (Fig. 5 and Table S5). Among the 1,689 DEGs in RHR, 54 significant GO terms were detected. However, no significant GO terms were observed among the 707 DEGs in SHR. In CHR, 30 significant GO terms were detected. In the CHR group, eight significant GO terms were observed in the biological process category, including response to stimulus, response to temperature stimulus, and response to heat; $17 \mathrm{GO}$ terms were in the cellular component category; and two significant GO terms were in the molecular function category. In the RHR group, 30, 14 and 10 significant GO terms were in the biological process, cellular component, and molecular function 
246

247

248

249

250

251

252

253

254

255

256

257

258

259

260

261

262

263

264

265

266

267

268

269

270

271

272

273

274

275

276

277

278

279

280

281

categories, respectively. The most significant GO terms, in decreasing order, were RNA biosynthetic process, nucleus, and DNA binding. In the molecular function category, 50 DEGs were specifically assigned to DNA-binding transcription factor activity, which may play an important role in heat stress tolerance.

The 50 DEGs of DNA-binding transcription factor activity could be divided into 11 transcription factor (TF) families, including HSF (1), WRKY (6), MADS (12), HD-ZIP (7), GATA (3), ERF (12), ABAI (1), b-ZIP (4), ARR-B (2), E2F (1), and NF-YA (1). Expression of the genes BGIOSGA006348 of HSF, BGIOSGA010835 of ABAI, BGIOSGA010142 of HAP, and $B G I O S G A 000303$ and BGIOSGA000304 of ARR-B was significantly upregulated. In addition, five genes in WRKY, eight genes in MADS, two genes in HD-ZIP, two genes in GATA, six genes in ERF, and two genes in b-ZIP were also upregulated (Table S6). These results suggest that, these $30 \mathrm{TF}$ genes may play important roles in heat stress resistance.

\section{Analysis of KEGG pathway enrichment}

In the KEGG analysis, 1,158 DEGs were classified into 225, 191, 239, and 211 functional pathways in HHZ_32 vs HHZ_40; 838 DEGs in IR36_32 vs IR36_40; 732 DEGs in IR36_40 vs HHZ_40; and 539 DEGs in IR36_32 vs HHZ_32. A total of 79 pathways were significant ( $P$-value $<0.05$ ) (Fig. 6). Among these pathways, the phenylpropanoid biosynthesis pathway was common in HHZ_32 vs HHZ_40, IR36_32 vs IR36_40, and IR36_40 vs HHZ_40, which suggests that heat stress impaired phenylpropanoid biosynthesis.

Based on further analysis of the three categories with different heat-stress responses, 146 DEGs in RHR were involved in 15 overrepresented pathways, including purine metabolism, pyrimidine metabolism, and amino sugar and nucleotide sugar metabolism; 45 DEGs in SHR were involved in 11 overrepresented pathways, including arginine biosynthesis, starch and sucrose metabolism, and polyketide sugar unit biosynthesis; and 184 DEGs in CHR were involved in 29 overrepresented pathways (Fig. 7 and Table S7).

A previous study showed that plant hormones are important for panicle development. Among the 15 KEGG pathways in RHR, 21 DEGs were involved in plant hormone signal transduction, of which 14 DEGs were upregulated in HHZ; three DEGs were involved in cytochrome P450 metabolism, which plays a role in brassinosteroid (BR) biosynthesis; and two were upregulated (Table 4).

In SHR and CHR, there were three common pathways: the starch and sucrose metabolism pathway, the NOD-like receptor signaling pathway, and the estrogen signaling pathway. Carbohydrate accumulation was essential for panicle development. In the KEGG analysis, seven DEGs involved in starch and sucrose metabolism were observed in SHR and 18 DEGs involved in starch and sucrose metabolism were observed in CHR. In SHR, the genes in HHZ were not 
282 different between HHZ_40 and HHZ_32. However, genes BGIOSGA010570 and 283 BGIOSGA026140 encoding sucrose synthase (EC 2.4.1.13), genes BGIOSGA026976, 284 BGIOSGA009181, and BGIOSGA030796 encoding trehalose-6-phosphate synthase (EC 2.4.1.15), 285 and gene BGIOSGA000509 encoding trehalose-6-phosphate phosphatase (EC 3.1.3.12) were 286 significantly downregulated in IR36_40 compared with IR36_32. However, gene BGIOSGA031385 encoding beta-amylase (EC 3.2.1.2) was significantly upregulated in IR36_40 compared with IR36_32 (Table 5).

\section{qRT-PCR verification}

To confirm the accuracy of the RNA-Seq results, ten representative DEGs each from the HHZ_32 vs HHZ_40 (a) and IR36_32 vs IR36_40 (b) groups, as well as five DEGs each from the IR36_40 vs HHZ_40 (c) and IR36_32 vs HHZ_32 (d) groups were chosen to determine relative expression. Of the ten DEGs from the HHZ_32 vs HHZ_40 group, five were in RHR: BGIOSGA022020 is related to BR synthesis, BGIOSGA006348 encodes a heat shock factor (Hsf), $B G I O S G A 017088$ is involved in the ETH TF family, BGIOSGA006285 participates in ethylene responsive regulation, and BGIOSGA024710 is an auxin-responsive gene involved in plant hormone transduction. Among the ten DEGs from the IR36_32 vs IR36_40 group, five were in SHR and encoded cytokinin oxidase/dehydrogenase (BGIOSGA005140), sucrose synthase (BGIOSGA026140), trehalose-6-phosphate synthase (BGIOSGA026970), trehalose-6-phosphate phosphatase (BGIOSGA000509), and catalase (BGIOSGA007252). Four DEGs were in CHR from the HHZ_32 vs HHZ_40 and IR36_32 vs IR36_40 groups and two common genes, BGIOSGA032653 and BGIOSGA015767, were validated. BGIOSGA032653 is involved in phenylpropanoid biosynthesis and BGIOSGA015676 encodes a heat shock protein (HSP). The qRT-PCR results for the DEGs were all consistent with the RNA-Seq data (Fig.8). differentiation inhibited panicle initiation and reduced spikelet number per panicle (Fig. 1).

\section{Discussion}

The exposure of rice plants to high temperature growing conditions during spikelet Previous studies have shown that the genes SP1, ASP1, TUT1, PAA2, and OSALMT7 are closely related to branch and spikelet development in rice (Bai et al. 2015; Heng et al. 2018; Li et al. 2010). However, in the current study, we observed no significant difference in the expression of these genes between the $40^{\circ} \mathrm{C}$ treatment and the $32^{\circ} \mathrm{C}$ control treatment in either rice cultivar, which indicates that the expression of these genes might not be inhibited in young panicles exposed to high temperature.

In general, the upregulation of HSPs contributes to the heat stress response in plants (Guan et 
317 al. 2010; Jagadish et al. 2010; Jung et al. 2013). Moon et al. (2014) reported that heterologous 318 overexpression of OsHSP1 (BGIOSGA015767, encoding a HSP) increased heat tolerance in 319 Arabidopsis. However, in the current study, BGIOSGA15767 expression was upregulated in both $320 \mathrm{HHZ}\left(\log 2\left(\mathrm{HHZ} \_40 / \mathrm{HHZ} \_32\right)=5.7, P\right.$-value $\left.=0\right)$ and IR36 $(\log 2($ IR36_40/IR36_32 $)=5.0, P$ value $=0$ ). In addition, there was no gene expression difference in the GO term of HSPs between cultivars, which demonstrates that the heat stress reaction is common to both rice cultivars when exposed to high temperature. The GO enrichment analysis revealed that the DEGs for the CHR group were commonly enriched in response to GO terms representing heat, stress, and temperature stimuli in the biological process category (Fig. 5). These results demonstrate that the heat stress response did not directly inhibit panicle development but rather may disrupt physiological processes related to panicle development.

An important factor determining heat tolerance is antioxidant capacity (Lan et al. 2016). Buer et al. (2010) reported that flavonoids can positively regulate reactive oxygen species (ROS), which can affect the transport of plant hormones and influence pollen development. The flavonoid synthesis pathway was overrepresented in the IR36_32 vs IR36_40 group. Specifically, five genes involved in flavonoid synthesis were downregulated at $40^{\circ} \mathrm{C}$, which might indicate a reduction in the antioxidant capacity of IR36 under heat stress. In addition, 14 DEGs in the IR36_32 vs IR36_40 group were enriched in the peroxisome pathway. Among these, 10 DEGs were significantly downregulated and four DEGs were significantly upregulated. However, the peroxisome pathway was not significant in the KEGG analysis of HHZ_32 vs HHZ_40 (Fig. 6). BGIOSGA007252 and BGIOSGA011520, which encode catalase (EC:1.11.1.6), were significantly downregulated in IR36 at $40^{\circ} \mathrm{C}$ compared with $32^{\circ} \mathrm{C}$, whereas no expression differences were observed in $\mathrm{HHZ} 32$ vs $\mathrm{HHZ}_{4} 40$. This finding suggests that high temperature had a greater negative effect on the antioxidant capacity of IR36 than of HHZ, which provides a primary explanation for the greater heat injury observed in the young IR36 panicles than in those of HHZ.

Regulation of endogenous hormones affects the development of young panicles. Wu et al. (2017) reported that a lower spikelet number under high temperature growing conditions was associated with cytokinin degradation. In the current study, BGIOSGA001314, which encodes a cytokinin-activity enzyme, did not differ between the $40^{\circ} \mathrm{C}$ and $32^{\circ} \mathrm{C}$ treatments in $\mathrm{HHZ}(\log 2$ $($ HHZ_40/HHZ_32 $)=-0.41)$ or IR36 $(\log 2($ IR36_40/IR36_32 $)=-0.38)$. However, the gene BGIOSGA005140, which encodes cytokinin oxidase/dehydrogenase, was significantly upregulated in the IR36_32 vs IR36_40 group ( $\log 2$ fold change $=1.67$, $P$-value $=0.004)$, but was not different in the HHZ_32 vs HHZ_40 group ( $\log 2$ fold change $=0.86, P$-value $=0.088)$. These results are consistent with those of $\mathrm{Wu}$ et al. (2016) and suggest that spikelet formation is associated with cytokinin degradation and greater degradation occurs at high temperatures in the heat-susceptible cultivar than in the heat-tolerant cultivar. 
353

354

355

356

357

358

359

360

361

362

363

364

365

366

367

368

369

370

371

372

373

374

375

376

377

378

379

380

381

382

383

384

385

386

387

388

The DEGs in RHR were enriched in 54 GO terms (Fig. 5). The GO term analysis revealed biological processes promoting resistance to heat stress in the heat-tolerant cultivar HHZ. Downregulation of BGIOSGA022020 in the heterocycle biosynthetic process induces GRAS protein reduction, which promotes BR synthesis to enhance heat tolerance (Vriet et al. 2012). In the molecular function category for RHR, 50 DEGs were involved in DNA-binding transcription factor activity. BGIOSGA006348 encoded an HSF TF and was upregulated in the HHZ_32 vs HHZ_40 group, although differences were not observed in the IR36_32 vs IR36_40 group. Wang et al. (2009) reported that the higher expression of heat shock TFs contributed to high temperature tolerance. WRKY genes encode TFs that play important roles in abiotic stress responses (Chen et al. 2010), especially to abscisic acid (ABA) (Zhen et al. 2005). In this study, six DEGs were WRKY TFs, namely, BGIOSGA003134, BGIOSGA017063, BGIOSGA029574, BGIOSGA005924, BGIOSGA024948, and BGIOSGA033505, which might promote young panicle development associated with sucrose consumption mediated by ABA under high temperature (Feng et al. 2018). However, few studies have reported the relationship between the WRKY family and heat resistance, which should be further studied. BGIOSGA029574 is a general stress-response gene, which has putative functions in distinct cellular processes, such as transcription regulation, stress response, and sugar metabolism under Fe-excess-induced, dark-induced, and droughtinduced stress (Ricachenevsky et al. 2010). Among the six WRKY genes, BGIOSGA017063 was downregulated while the other five genes were upregulated, although the gene has not been cloned for the gene function analysis and therefore requires further study. Of the 10 DEGs in the ETH family, five genes were downregulated and the downregulation of BGIOSGA017088 reduced the ABA content and promoted gibberellin (GA) signal transduction, which is beneficial for rice plant growth (Yaish et al. 2010). The upregulation of BGIOSGA006285, BGIOSGA010867, BGIOSGA030019, BGIOSGA005915, and BGIOSGA012535 plays an important role in ethylene response regulation. Cao et al. (2006) reported that the upregulation of BGIOSGA005915 enhanced tolerance to salt, cold, drought, and wounding, and the current study reveals that this gene might also contribute to the improvement of high-temperature stress resistance. BGIOSGA000303 and BGIOSGA000304 are genes in the cytokinin receptor family, and the upregulation of these two genes promotes cytokinin activation (Ito \& Kurata 2006). The MADs box gene is related to flower development (Kobayashi et al. 2012) and the upregulation of the MAD genes in RHR indicated that the MAD family might enhance heat stress tolerance. The HZZIP TF family might have a similar function.

In the RHR category, the DEGs enriched in the KEGG pathways appear beneficial for heatstress tolerance, including plant hormone signal transduction and BR biosynthesis. Twenty-one DEGs were involved in plant hormone signal transduction, of which 14 DEGs were upregulated, including the auxin-responsive genes BGIOSGA024710, BGIOSGA001585, BGIOSGA019301, 
and BGIOSGA037837, which facilitate rice plant growth (Hagen \& Guilfoyle 2002). In BR biosynthesis, BGIOSGA002945, which encodes D2/CYP90D2, a gene that catalyzes the steps from 6-deoxoteasterone to 3-dehydro-6-deoxoteasterone and from teasterone to 3-dehydroteasterone, was upregulated to promote BR synthesis in the latter pathway (Hong et al. 2003), and BGIOSGA001585 was downregulated to promote BR activity (Sakamoto et al. 2011). The genes related to hormone signal transduction and BR biosynthesis might contribute to young panicle development under high temperature. Seven DEGs involved in plant hormone signal transduction were downregulated, and among these, BGIOSGA036617, BGIOSGA034767, and BGIOSGA010559 have not been cloned for functional analysis while BGIOSGA034772 plays a more important role in organismal development. The genes BGIOSGA024374, BGIOSGA023368, BGIOSGA000304 and BGIOSGA005312 are A-type response regulated genes (Jain et al. 2006). However, it is unclear whether the downregulation of BGIOSGA024374 and BGIOSGA023368 contributes to improved heat tolerance in rice varieties. In addition, the downregulated gene, BGIOSGA010919, is an ABA receptor. Tian et al. (2015) reported that ABA accumulation upregulates gene expression. In the current study, downregulation of BGIOSGA010919 may contribute to excessive $\mathrm{ABA}$ accumulation. The role of $\mathrm{ABA}$ in panicle development requires further study. BGIOSGA014915, which participates in BR synthesis, was downregulated in RHR. Previous reports have found that BRs can modulate the metabolic responses of plants to abiotic environmental stresses (Vriet et al. 2012; Wang et al. 2018). BR accumulation reportedly reduces spikelet degeneration under nitrogen application (Zhang, 2018). BGIOSGA002945 and BGIOSGA014915 participate in different BR biosynthesis pathways (Shi, 2015), but BGIOSGA002945 may play a more important role in modulating spikelet development under high temperature.

Carbohydrate storage and utilization are essential for panicle initiation (Tian et al. 2016). The KEGG analysis showed that the phenylpropanoid biosynthesis pathway was commonly overrepresented in HHZ_32 vs HHZ_40, IR36_32 vs IR36_40, and IR36_40 vs HHZ_40. The phenylpropanoid biosynthesis pathway is involved in lignin synthesis, which suggests that high temperature inhibits lignin synthesis; however, phenylpropanoid biosynthesis was not associated with heat tolerance in our heat resistant cultivar (Fig. 6). In the SHR category, seven DEGs were enriched in the starch and sucrose metabolism pathway (Fig.7b). The gene BGIOSGA031385, which encodes beta-amylase, was significantly upregulated in IR36_32 vs IR36_40, suggesting that it promoted starch hydrolysis and reduced carbohydrate storage. The genes BGIOSGA010570 and BGIOSGA026140, which encode sucrose synthesis, were significantly downregulated in the IR36_32 vs IR36_40 group, whereas no difference in expression was observed in the HHZ_32 vs HHZ_40 group. Sucrose degrades into uridine 5'-diphosphoglucose and fructose, which are major forms of carbon used for energy. Impairment of sucrose synthase activity reportedly reduced 
425 resistance to heat stress (Hirose et al. 2008; Takehara et al. 2018). The results of the current study 426 suggest that impaired carbohydrate metabolism in the heat-susceptible cultivar aggravated spikelet 427 reduction. The starch and sucrose pathway genes were also highly represented in the CHR group 428 (Fig.7c). Such genes are involved in the downregulation of genes encoding beta429 fructofuranosidase, fructokinase, beta-glucosidase, trehalose-6-phosphate phosphatase, alphatrehalase, and others. Trehalose-6-phosphate synthase, trehalose-6-phosphate phosphatase, and alpha-trehalase are involved in trehalose synthesis. Trehalose plays an important role in abiotic stress resistance, and trehalose-6-phosphate, an intermediate product of trehalose synthesis participates in sucrose signal transduction (Lunn et al. 2006; Ruan 2014). Nunes et al. (2013) reported that trehalose-6-phosphate served as a sugar signal that could induce the expression of genes associated with the alleviation of abiotic stress injury. In this study, certain DEGs in the CHR group were also upregulated to promote trehalose-6-phosphate synthesis, and the upregulation of BGIOSGA026976, BGIOSGA009181, and BGIOSGA030796 promoted trehalose6-phosphate synthesis in SHR. These findings indicate that trehalose-6-phosphate synthesis may be a normal response of young rice panicles to high temperature and that the heat-sensitive rice cultivar synthesizes trehalose-6-phosphate more readily than the heat-tolerant cultivar in response to heat stress. However, the gene encoding trehalose-6-phosphate phosphatase, BGIOSGA000509, was significantly downregulated in IR 36 at $40^{\circ} \mathrm{C}$ compared with that at $32^{\circ} \mathrm{C}$, which might cause a decrease in trehalose content and in turn disrupt carbohydrate distribution. Our results suggest that trehalose-6-phosphate metabolism was disordered under the high temperature condition and that the effects were more severe in the heat-susceptible cultivar than in the heat-tolerant cultivar.

A close relationship is observed between endogenous hormones and carbohydrate

\section{Conclusions}

In summary, heat stress-responsive DEGs in young panicles were identified by a transcriptome analysis of a heat-tolerant rice cultivar and a heat-susceptible rice cultivar grown at high temperature $\left(40^{\circ} \mathrm{C}\right)$ and a control temperature $\left(32^{\circ} \mathrm{C}\right)$. The statistical analysis of 5,533 DEGs revealed three categories of genes (RHR, SHR, and CHR) containing a total of 4,070 DEGs. We 
460

461

462

463

464

465

466

467

468

469

470

471

472

473

474

475

476

477

478

479

480

481

482

483

484

485

486

487

488

489

490

491

492

493

494

495

496

497

498

enriched in the RHR category as well as the differential expression of genes involved in the starch and sucrose metabolism pathway that were overrepresented in the SHR category. Overall, DEGs related to plant hormones and signal transduction might be specifically beneficial for young panicle development at high temperature. Heat-tolerant cultivars seem to increase endogenous hormones and maintain a stable carbohydrate metabolism pathway under high temperature. However, certain metabolic pathways, including starch and sucrose metabolism, were much more damaged in the heat susceptible cultivars under high temperatures, and this damage might have inhibited the of panicle development.

\section{References}

Anand RY, Kumar SD, and Narayan TK. 2018. Effect of irrigation method on adaptation capacity of rice to climate change in subtropical India. International Journal of Plant Production, 2018, 12: 203-217.

Bai JT, Zhu XD, Wang Q, Zhang J, Chen HQ, Dong GJ, Zhu L, Zheng HK, Xie QJ, Nian JQ, Chen F, Fu Y, Qian Q, and Zuo JR. 2015. Rice TUTOU1 encodes a SCAR-like protein that is important for actin organization and panicle development. Plant Physiology, 169:1179-1191.

Buer CS, Imin N, and Djordjevic MA. 2010. Flavonoids: new roles for old molecules. Journal of Integrative Plant Biology, 52:98-111.

Cao YY, Duan H, Yang LN, Wang ZQ, Zhou SC, and Yang JC. 2009. Effect of heat-stress during meiosis on grain yield of rice cultivars differing in heat-tolerance and its physiological mechanism. Acta Agronomica Sinica, 34:2134-2142.

Cao Y, Song F, Goodman RM, and Zheng Z. 2006. Molecular characterization of four rice genes encoding ethyleneresponsive transcriptional factors and their expressions in response to biotic and abiotic stress. Journal of Plant Physiology, 163:1167-1178.

Chen H, Lai ZB, Shi JW, Xiao Y, Chen ZX, and Xu XP. 2010. Roles of Arabidopsis WRKY18, WRKY40 and WRKY60 transcription factors in plant responses to abscisic acid and abiotic stress. BMC Plant Biology, 10:1-15.

Czechowski T, Bari RP, Stitt M, Scheible WR, and Udvardi MK. 2004. Real-time RT-PCR profiling of over 1400 Arabidopsis transcription factors: unprecedented sensitivity reveals novel root- and shoot-specific genes. Plant Journal 38:366-379.

Ding C, Wang S, and Ding Y. 2016. Functional genes of panicle development in response to nitrogen fertilizer in rice. Chinese Bullrtin of Botany, 51:488-498.

Dwivedi SK, Kumar S, Prakash V, Mondal S, and Mishra JS. 2015. Influence of rising atmospheric $\mathrm{CO}_{2}$ concentrations and temperature on morpho-physiological traits and yield of rice genotypes in sub humid climate of eastern India. American Journal of Plant Sciences, 6:2239-2249.

Endo M, Tsuchiya T, Hamada K, Kawamura S, Yano K, Ohshima M, Higashitani A, Watanabe M, and KawagishiKobayashi M. 2009. High temperatures cause male sterility in rice plants with transcriptional alterations during pollen development. Plant Cell Physiology, 50:1911-1922.

Espe MB, Hill JE, Hijmans RJ, McKenzie K, Mutters R, Espino LA, Leinfelder-Miles M, van Kessel C, and Linquist BA. 2017. Point stresses during reproductive stage rather than warming seasonal temperature determine yield 
in temperate rice. Global Change Biology, 23:4386-4395.

Fang XW, Tang LH, and Wang YP. 2006. Selection on rice germplasm tolerant to high temperature. Journal of Plant Genetic Resources, 7:342-344.

Feng BH, Zhang CX, Chen TT, Zhang XF, Tao LX, and Fu GF. 2018. Salicylic acid reverses pollen abortion of rice caused by heat stress. BMC Plant Biology, 18: 245.

Guan JC, Yeh CH, Lin YP, Ke YT, Chen MT, You JW, Liu YH, Lu CA, Wu SJ, and Lin CY. 2010. A 9 bp ciselement in the promoters of class I small heat shock protein genes on chromosome 3 in rice mediates Lazetidine-2-carboxylic acid and heat shock responses. Journal of Exprimental Botany, 61:4249-4261.

Hagen G, and Guilfoyle T. 2002. Auxin-responsive gene expression: genes, promoters and regulatory factors. Plant Molecular Biology, 49:373-385.

Heng YQ, Wu CY, Long Y, Luo S, Ma J, Chen J, Liu JF, Zhang H, Ren YL, Wang M, Tan JJ, Zhu SS, Wang JL, Lei CL, Zhang X, Guo XP, Wang HY, Cheng ZJ, and Wan JM. 2018. OsALMT7 maintains panicle size and grain yield in rice by mediating malate transport. The Plant Cell, 30:889-906.

Hirose T, Scofield GN, and Terao T. 2008. An expression analysis profile for the entire sucrose synthase gene family in rice. Plant Science, 174:534-543.

Hong Z, Ueguchitanaka M, Umemura K, Uozu S, Fujioka S, Takatsuto S, Yoshida S, Ashikari M, Kitano H, and Matsuoka M. 2003. A rice brassinosteroid-deficient mutant, ebisu dwarf(d2), is caused by a loss of function of a new member of cytochrome P450. The Plant Cell, 15:2900-2910.

Islam MR, Feng BH, Chen TT, Fu WM, Zhang CX, Tao LX, and Fu GF. 2018. Abscisic acid prevents pollen abortion under high temperature stress by mediating sugar metabolism in rice spikelets. Physiologia Plantarum, 2019, 165(3): 644-663.

Ito Y, and Kurata N. 2006. Identification and characterization of cytokinin-signalling gene families in rice. Gene, 382:57-65.

Jagadish SV, Muthurajan R, Oane R, Wheeler TR, Heuer S, Bennett J, and Craufurd PQ. 2010. Physiological and proteomic approaches to address heat tolerance during anthesis in rice (Oryza sativa L.). Journal of Expermental Botany, 61:143-156.

Jain M, Tyagi AK, and Khurana JP. 2006. Molecular characterization and differential expression of cytokininresponsive type-A response regulators in rice (Oryza sativa). BMC Plant Biology, 6:1-11.

Jung KH, Gho HJ, Nguyen MX, Kim SR, and An G. 2013. Genome-wide expression analysis of HSP70 family genes in rice and identification of a cytosolic $H S P 70$ gene highly induced under heat stress. Functional \& Integrative Genomics, 13:391-402.

Kang HG, Jang S, Chung JE, Cho YG, and An G. 2013. Characterization of two rice MADS box genes that control flowering time. Molecules Cells, 7:559-566.

Kim J, Shon J, Lee CK, Yang W, Yoon Y, Yang WH, Kim YG, and Lee BW. 2011. Relationship between grain filling duration and leaf senescence of temperate rice under high temperature. Field Crops Research, 122:207-213.

Kobayashi K, Yasuno N, Sato Y, Yoda M, Yamazaki R, Kimizu M, Yoshida H, Nagamura Y, and Kyozuka J. 2012. Inflorescence meristem identity in rice is specified by overlapping functions of three $A P 1 / F U L$-Like MADS Box Genes and PAP2, a SEPALLATA MADS Box Gene. The Plant Cell, 24:1848-1859.

Lan X, Gu ZD, Ding YF, Wang K, Jiang Q, and Zhu C. 2016. Effect of high temperature stress on physiological characteristics of spikelet of rice during flowering stage. Chinese Journal of Rice Science, 30:637-646.

Li SB, Qian Q, Fu ZM, Zeng DL, Meng XB, Kyozuka J, Maekawa M, Zhu XD, Zhang J, and Li JY. 2010. Short 
panicle1 encodes a putative PTR family transporter and determines rice panicle size. Plant Journal, 58:592605.

Liu XW, Meng YL, Zhou ZG, and Cao WX. 2005. Dynamic characteristics of floert differentiation and degeneration in rice. Acta Agronomica Ainica, 31: 451-455.

Lunn JE, Feil R, Hendriks JHM, Gibon Y, Morcuende R, Osuna D, Scheible W, Carillo P, Hajirezaei MR, and Stitt M. 2006. Sugar-induced increases in trehalose 6-phosphate are correlated with redox activation of ADPglucose pyrophosphorylase and higher rates of starch synthesis in Arabidopsis thaliana. Biochemical Journal, 397:139-148.

Min L, Li Y, Hu Q, Zhu L, Gao W, Wu Y, Ding Y, Liu S, Yang X, and Zhang X. 2014. Sugar and auxin signaling pathways respond to high-temperature stress during anther development as revealed by transcript profiling analysis in cotton. Plant Physiology, 164:1293-1308.

Moon JC, Ham DJ, Hwang SG, Yong CP, Lee C, and Jang CS. 2014. Molecular characterization of a heat inducible rice gene, OsHSP1, and implications for rice thermotolerance. Genes \& Genomics, 36:151-161.

Morita S, Wada H, and Matsue Y. 2016. Countermeasures for heat damage in rice grain quality under climate change. Plant Production Science, 19(1):1-11.

Nunes C, and Paul MJ. 2013. The trehalose 6-Phosphate/SnRK1 signaling pathway primes growth recovery following relief of sink limitation. Plant Physiology, 162:1720-1732.

Prasad PVV, Bheemanahalli R, and Jagadish SVK. 2017. Field crops and the fear of heat stress-Opportunities, challenges and future directions. Field Crops Research, 200:114-121.

Ricachenevsky FK, Sperotto RA, Menguer PK, Fett JP. 2010. Identification of Fe-excess-induced genes in rice shoots reveals a WRKY transcription factor responsive to Fe, drought and senescence. Molecular Biology Reports, 37:3735-3745.

Ruan YL. 2014. Sucrose metabolism: gateway to diverse carbon use and sugar signaling. Annual Review Plant Biology, 65:33-67.

Sakamoto T, Kawabe A, Tokidasegawa A, Shimizu B, Takatsuto S, Shimada Y, Fujioka S, and Mizutani M. 2011. Rice CYP734As function as multisubstrate and multifunctional enzymes in brassinosteroid catabolism. Plant Journal, 67:1-12.

Shi ZY. 2015. Fine mapping of grain width locus GW12 and functional verification of a grain length gene SG4 in Rice. Ph. D. dissertation, Chinese Academy of Agricultural Sciences, Beijing.

Stocher TF, Plattner GK, Tignor MMB, Allen SK, Boschung J, Nauel A, Xia Y, Bex V, and Midgley P. 2013. CLIMATE CHANGE 2013: The Physical Science Basis, working group I contribution to the fifth assessment report of the intergovernmental panel on climate change. Cambridge Vniversity Press, Cambridge, UK.

Takehara K, Murata K, Yamaguchi T, Yamaguchi K, Chaya G, Kido S, Iwasaki Y, Ogiwara H, Ebitani T, and Miura K. 2018. Thermo-responsive allele of sucrose synthase 3 (Sus3) provides high-temperature tolerance during the ripening stage in rice (Oryza sativa L.). Breeding Science, 63:336-342.

Tao FL, Zhang Z, Shi WJ, Liu YJ, Xiao DP, Zhang S, Zhu Z, Wang M, and Liu FS. 2013. Single rice growth period was prolonged by cultivars shifts, but yield was damaged by climate change during 1981-2009 in China, and late rice was just opposite. Global Change Biology, 19:3200-3209.

Tian QL, Liu B, Zhong XY, Zhao M, Sun H, and Ren WJ. 2016. Relationship of NSC with the formation of branches and spikelets and the yield traits of Indica hybrid rice in different planting methods. Scientia Agricultura Sinica 49:35-53. 
Tian XJ, Wang ZY, LiXF, Lv TX, Liu HZ, Wang LZ, Niu HB, Bu QY. Characterization and functional analysis of pyrabactin resistance-like abscisic acid receptor family in rice. Rice, 2015,8:28.

Vriet C, Russinova E, and Reuzeau C. 2012. Boosting crop yields with plant steroids. The Plant Cell, 24:842-857.

Wang C, Qian Z, and Shou HX. 2009. Identification and expression analysis of OsHsfs in rice. Journal of Zhejiang University-Science B(Biomedicine \& Biotechnology), 10:291-300.

Wang YL, Wang L, Zhou JX, Hu SB, Chen HZ, Xiang J, Zhang YK, Zeng YJ, Shi QH, Zhu DF, and Zhang YP. Research progress on heat stress of rice at flowering stage. Rice Science, 2019, 26(1): 1-10.

Wang YL, Zhang YP, Xiang J, Wang L, Chen HZ, Zhang YK, Zhang WQ, and Zhu F. 2017. Response of indica rice spikelet differentiation and degeneration to air temperature and solar radiation of different sowing dates. Chinese Journal of Applied Ecology, 28:3571-3580.

Wang ZQ, Zhang WY, and Yang JC. 2018. Physiological mechanism underlying spikelet degeneration in rice. Journal of Integrative Agriculture, 17:1475-1481.

Wu C, Cui KH, Wang WC, Li Q, Fahad S, Hu QQ, Huang JL, Nie LX, Mohapatra PK, and Peng SB. 2017. Heatinduced cytokinin transportation and degradation are associated with reduced panicle cytokinin expression and fewer spikelets per panicle in rice. Frontiers in Plant Science, 8:371.

Wu C, Cui KH, Wang WC, Li Q, Fahad S, Hu QQ, Huang JL, Nie LX, and Peng SB. 2016. Heat-induced phytohormone changes are associated with disrupted early reproductive development and reduced yield in rice. Scientific Reports, 6:34978.

Yaish MW, Elkereamy A, Zhu T, Beatty PH, Good AG, Bi YM, and Rothstein SJ. 2010. The APETALA-2-like transcription factor OSAP2-39 controls key interactions between abscisic acid and gibberellin in rice. Plos Genetics, 6:e1001098.

Yamagishi J, Miyamoto N, Hirotsu S, Laza RC, and Nemoto K. 2004. QTLs for branching, floret formation, and preflowering floret abortion of rice panicle in a temperate japonica $\mathrm{x}$ tropical japonica cross. Theoretical \& Applied Genetics, 109:1555-1561.

Yamakawa H, and Hakata M. 2010. Atlas of rice grain filling-related metabolism under high temperature: joint analysis of metabolome and transcriptome demonstrated inhibition of starch accumulation and induction of amino acid accumulation. Plant \& Cell Physiology, 51:795-809.

Yamakawa H, Hirose T, Kuroda M, and Yamaguchi T. 2007. Comprehensive expression profiling of rice grain fillingrelated genes under high temperature using DNA microarray. Plant Physiology, 144:258-277.

Zhen X, Zhang ZL, Zou XL, Huang J, Ruas P, Thompson D, and Shen QJ. 2005. Annotations and Functional Analyses of the Rice WRKY Gene Superfamily Reveal Positive and Negative Regulators of Abscisic Acid Signaling in Aleurone Cells[J]. Plant Physiology, 137: 176-189.

Zhong LJ, Cheng FM, Wen X, Sun ZX, and Zhang GP. 2010. The deterioration of eating and cooking quality caused by high temperature during grain filling in early-season indica rice cultivars. Journal of Agronomy \& Crop Science, 191:218-225.

Zhou SC, Li H, Huang D, Lu D, Lai Z, Zhou D, Li K, Wang C, and Li H. 2012. Breeding and application of Huanghuazhan-A new variety with 1st class rice quality of mational standard. Hubei Agricultural Sciences, 51:1960-1964.

Zhang WY. 2018. Mechanism underlying water and nitrogen regulation spikelet development and grain filling of rice. Ph.D. dissertation, Yangzhou university, Yangzhou.

Peer) reviewing PDF | (2018:12:33492:5:0:NEW 21 Jul 2019) 
Figure legends:

624

Figure 2. Gene expression in the four comparison groups. (a) HHZ_32 vs HHZ_40, (b) IR36_32

Figure 4. Enriched GO terms $(P<0.05)$ of all DEGs. (a) biological process, (b) cellular component, and (c) molecular function.

Figure 5. Enriched GO terms $(P<0.05)$ of the DEGs in the RHR and CHR groups. (a) biological process, (b) cellular component, and (c) molecular function.

Figure 6. KEGG enrichment analysis of all DEGs. (a) HHZ_32 vs HHZ_40, (b) IR36_32 vs IR36_40, (c) IR36_40 vs HHZ_40, and (d) IR36_32 vs HHZ_32.

Figure 7. KEGG enrichment analysis for heat stress responsive genes from the three categories. (a) RHR, (b) SHR, and (c) CHR.

Figure 8. Gene expression levels determined by RNA-Seq and qRT-PCR. (a) HHZ_32 vs HHZ_40, (b) IR36_32 vs IR36_40, (c) IR36_40 vs HHZ_40, and (d) IR36_32 vs HHZ_32. 
Table $\mathbf{1}$ (on next page)

Panicle characters after high temperature treatment 
1 Table 1 Panicle characters after high temperature treatment

\begin{tabular}{|c|c|c|c|c|c|c|c|c|c|}
\hline $\begin{array}{c}\text { Cultivar } \\
\text { s }\end{array}$ & $\begin{array}{c}\text { Treatme } \\
\mathrm{nt}\end{array}$ & $\begin{array}{c}\text { Panicle } \\
\text { weight(g) }\end{array}$ & $\begin{array}{l}\text { Spikelet } \\
\text { number }\end{array}$ & $\begin{array}{c}\text { The number of } \\
\text { differentiated } \\
\text { spikelet }\end{array}$ & $\begin{array}{c}\text { The proportion of } \\
\text { degenerated } \\
\text { spikelet }(\%)\end{array}$ & $\begin{array}{c}\text { Spikelet } \\
\text { fertility (\%) }\end{array}$ & $\begin{array}{c}\text { Grain } \\
\text { weight }(\mathrm{mg})\end{array}$ & $\begin{array}{l}\text { Glume } \\
\text { length } \\
(\mathrm{mm})\end{array}$ & $\begin{array}{l}\text { Glume } \\
\text { width } \\
(\mathrm{mm})\end{array}$ \\
\hline \multirow[t]{3}{*}{$\mathrm{HHZ}$} & $32^{\circ} \mathrm{C}$ & $3.6 \pm 0.4$ & $235.0 \pm 20.0$ & $335.3 \pm 20.5$ & $30.0 \pm 1.7$ & $83.0 \pm 2.0$ & $18.5 \pm 0.3$ & $8.7 \pm 0.2$ & $2.5 \pm 0.0$ \\
\hline & & & $182.7 \pm 11.2^{*}$ & & & & & & \\
\hline & $40^{\circ} \mathrm{C}$ & $2.4 \pm 0.3^{* *}$ & $*$ & $303.3 \pm 10.1$ & $39.7 \pm 5.3$ & $78.5 \pm 1.3^{* *}$ & $17.0 \pm 0.3^{* *}$ & $7.8 \pm 0.1^{* *}$ & $2.2 \pm 0.1^{* *}$ \\
\hline \multirow[t]{2}{*}{ IR36 } & $32^{\circ} \mathrm{C}$ & $3.1 \pm 0.4$ & $183.3 \pm 7.6$ & $264.7 \pm 13.8$ & $30.7 \pm 1.5$ & $81.5 \pm 1.8$ & $20.5 \pm 0.3$ & $8.1 \pm 0.3$ & $2.5 \pm 0.0$ \\
\hline & $40^{\circ} \mathrm{C}$ & $1.0 \pm 0.1^{* *}$ & $85.0 \pm 13.5^{* *}$ & $176.7 \pm 17.6^{* *}$ & $51.4 \pm 9.3^{* *}$ & $73.5 \pm 1.1^{* *}$ & $16.0 \pm 0.1^{* *}$ & $6.8 \pm 0.2^{* *}$ & $2.3 \pm 0.1^{* *}$ \\
\hline
\end{tabular}

2

* and $* *$ indicate significance differences between the control $\left(32^{\circ} \mathrm{C}\right)$ ang high $\left({ }^{\circ} \mathrm{C}\right)$ temperature treatments (one-tailed Student's t-test): ${ }^{*} P<0.05 ;{ }^{* *} P<0.01$. 
Table 2 (on next page)

Table 2. RNA sequencing results 
1

2

3

\begin{tabular}{ccccc}
\hline Sample & HHZ_32 & HHZ_40 & IR36_32 & IR36_40 \\
\hline Raw reads & 44231722 & 45513241 & 45877838 & 46465046 \\
Clean reads & 44032896 & 45256701 & 45580821 & 46252929 \\
& $(99.6 \%)$ & $(99.4 \%)$ & $(99.4 \%)$ & $(99.5 \%)$ \\
Total mapped & 38834391 & 39148950 & 39541858 & 40418126 \\
& $(87.8 \%)$ & $(86.0 \%)$ & $(86.2 \%)$ & $(87.0 \%)$ \\
Uniquely mapped & 37502957 & 37759013 & 38120438 & 38853775 \\
& $(84.8 \%)$ & $(83.0 \%)$ & $(83.1 \%)$ & $(83.1 \%)$ \\
Multiply mapped & 1331434 & 1389937 & 1421421 & 1561018 \\
& $(3.0 \%)$ & $(3.1 \%)$ & $(3.1 \%)$ & $(3.6 \%)$ \\
\hline
\end{tabular}

4 Note: HHZ_32: The sample of HHZ treated with $32^{\circ} \mathrm{C}$; HHZ_40: The sample of HHZ treated with $40^{\circ} \mathrm{C}$; IR36_32:

5 The sample of IR 36 treated with $32^{\circ} \mathrm{C}$; IR36_40: The sample of IR 36 treated with $40^{\circ} \mathrm{C}$.

6

7 
Table 3 (on next page)

Table 3. Classification of three categories of DEGs 
1

2

3

4

Table 3. Classification of three categories of DEGs.

\begin{tabular}{cll}
\hline Categories & Subgroups & Number of DEGs \\
\hline RHR & Only HHZ_32 vs HHZ_40 & 1157 \\
& HHZ_32 vs HHZ_40 $\cap$ IR36_40 vs HHZ_40 & 531 \\
SHR & Only IR36_32 vs IR36_40 & 603 \\
& IR36_32 vs IR36_40 $\cap$ IR36_40 vs HHZ_40 & 104 \\
CHR & Only IR36_40 vs HHZ_40 & 524 \\
& HHZ_32 vs HHZ_40 $\cap$ IR36_32 vs IR36_40, & \\
& HHZ_32 vs HHZ_40 $\cap$ IR36_32 vs IR36_40 $\cap$ IR36_40 vs HHZ_40 & 1151
\end{tabular}

5 Note: RHR, heat-resistant-cultivar-related genes; SHR, heat-susceptible-cultivar-related genes;

6 CHR, common heat stress-response genes. 


\section{Table 4 (on next page)}

Table 4. Gene expression of DEGs in plant hormone signal transduction and BR biosynthesis of RHR 
Table 4. Gene expression of DEGs in Plant hormone signal transduction and BR biosynthesis of RHR

\begin{tabular}{|c|c|c|c|c|c|c|c|}
\hline ID & Gene annotation & $\begin{array}{c}\text { Cultiva } \\
\text { r }\end{array}$ & baseMean & $32^{\circ} \mathrm{C}$ & $40^{\circ} \mathrm{C}$ & $\log 2$ FoldChange & pval \\
\hline \multirow[t]{2}{*}{ BGIOSGA018672 } & Pseudo histidine-containing phosphotransfer protein 2 & $\mathrm{HHZ}$ & 64.7 & 36.9 & 92.5 & 1.33 & 0.00 \\
\hline & & IR36 & 59.4 & 49.2 & 69.7 & 0.50 & 0.06 \\
\hline \multirow[t]{2}{*}{ BGIOSGA004140 } & Probable protein phosphatase $2 \mathrm{C} 8$ & $\mathrm{HHZ}$ & 665.2 & 219.8 & 1110.7 & 2.34 & 0.00 \\
\hline & & IR36 & 465.6 & 355.3 & 575.9 & 0.70 & 0.00 \\
\hline \multirow[t]{2}{*}{ BGIOSGA005312 } & Two-component response regulator ORR3 & HHZ & 50.6 & 28.6 & 72.6 & 1.35 & 0.00 \\
\hline & & IR36 & 26.3 & 24.2 & 28.5 & 0.24 & 0.58 \\
\hline \multirow[t]{2}{*}{ BGIOSGA024710 } & Auxin-responsive protein IAA24 & $\mathrm{HHZ}$ & 807.3 & 458.2 & 1156.3 & 1.34 & 0.00 \\
\hline & & IR36 & 828.1 & 653.4 & 1002.7 & 0.62 & 0.00 \\
\hline \multirow[t]{2}{*}{ BGIOSGA010835 } & ABSCISIC ACID-INSENSITIVE 5-like protein 2 & HHZ & 146.5 & 85.8 & 207.2 & 1.27 & 0.00 \\
\hline & & IR36 & 83.4 & 74.0 & 92.8 & 0.33 & 0.26 \\
\hline \multirow[t]{2}{*}{ BGIOSGA011032 } & Probable protein phosphatase $2 \mathrm{C} 30$ & $\mathrm{HHZ}$ & 102.9 & 53.4 & 152.4 & 1.51 & 0.00 \\
\hline & & IR36 & 113.6 & 108.0 & 119.3 & 0.14 & 0.69 \\
\hline \multirow[t]{2}{*}{ BGIOSGA015611 } & Probable protein phosphatase 2C 37 & HHZ & 86.1 & 44.3 & 127.8 & 1.53 & 0.00 \\
\hline & & IR36 & 75.8 & 52.0 & 99.6 & 0.94 & 0.00 \\
\hline \multirow[t]{2}{*}{ BGIOSGA019301 } & Auxin-responsive protein IAA16 & $\mathrm{HHZ}$ & 97.4 & 56.6 & 138.3 & 1.29 & 0.00 \\
\hline & & IR36 & 79.7 & 76.6 & 82.8 & 0.11 & 0.61 \\
\hline \multirow[t]{2}{*}{ BGIOSGA008704 } & Auxin-responsive protein SAUR36 & $\mathrm{HHZ}$ & 36.6 & 22.9 & 50.3 & 1.14 & 0.00 \\
\hline & & IR36 & 23.1 & 22.5 & 23.6 & 0.07 & 0.91 \\
\hline \multirow[t]{2}{*}{ BGIOSGA012535 } & ARATH Protein ETHYLENE INSENSITIVE 3 & $\mathrm{HHZ}$ & 2890.1 & 1268.4 & 4511.8 & 1.83 & 0.00 \\
\hline & & IR36 & 2293.2 & 1543.8 & 3042.6 & 0.98 & 0.00 \\
\hline \multirow[t]{2}{*}{ BGIOSGA037772 } & ARATH Transcription factor PIF1 & $\mathrm{HHZ}$ & 27.5 & 14.2 & 40.8 & 1.52 & 0.00 \\
\hline & & IR36 & 17.3 & 13.3 & 21.4 & 0.69 & 0.24 \\
\hline \multirow[t]{2}{*}{ BGIOSGA000304 } & Two-component response regulator ORR26 & $\mathrm{HHZ}$ & 143.8 & 92.5 & 195.0 & 1.08 & 0.00 \\
\hline & & IR36 & 110.4 & 85.9 & 134.9 & 0.65 & 0.00 \\
\hline
\end{tabular}


BGIOSGA004789

BGIOSGA037837

BGIOSGA024374

BGIOSGA036617

BGIOSGA034772

BGIOSGA010559

BGIOSGA010919

BGIOSGA023368

BGIOSGA034767

BGIOSGA002945

BGIOSGA014915

BGIOSGA001585
Probable protein phosphatase $2 \mathrm{C}$

Auxin-responsive protein SAUR72

Two-component response regulator ORR7

Transcription factor TGAL11

$\mathrm{BTB} / \mathrm{POZ}$ domain and ankyrin repeat-containing protein

$$
\text { NH5.1 }
$$

Protein TIFY 10a

Abscisic acid receptor PYL5

Two-component response regulator ORR25

$\mathrm{BTB} / \mathrm{POZ}$ domain and ankyrin repeat-containing protein

$$
\text { NH5.2 }
$$

$$
\text { Cytochrome P450 90D2 }
$$

Cytochrome P450 724B1

Cytochrome P450 734A6

\begin{tabular}{lccccc} 
HHZ & 522.2 & 301.9 & 742.5 & 1.30 & 0.00 \\
IR36 & 623.2 & 501.8 & 744.5 & 0.57 & 0.02 \\
HHZ & 3.5 & 1.0 & 6.0 & 2.55 & 0.04 \\
IR36 & 0.8 & 1.3 & 0.3 & -2.07 & 0.67 \\
HHZ & 17.8 & 29.5 & 6.2 & -2.26 & 0.00 \\
IR36 & 49.3 & 58.2 & 40.4 & -0.53 & 0.10 \\
HHZ & 308.0 & 423.3 & 192.8 & -1.13 & 0.00 \\
IR36 & 572.4 & 700.8 & 444.0 & -0.66 & 0.00 \\
HHZ & 1148.1 & 1629.2 & 667.0 & -1.29 & 0.00 \\
IR36 & 1335.2 & 1698.8 & 971.6 & -0.81 & 0.00 \\
& & & & & \\
HHZ & 339.2 & 465.1 & 213.4 & -1.12 & 0.00 \\
IR36 & 374.0 & 492.0 & 256.0 & -0.94 & 0.00 \\
HHZ & 34.8 & 55.0 & 14.5 & -1.92 & 0.00 \\
IR36 & 52.9 & 58.0 & 47.8 & -0.28 & 0.33 \\
HHZ & 4.4 & 8.8 & 0.0 & - Inf & 0.00 \\
IR36 & 3.2 & 5.5 & 1.0 & -2.49 & 0.15 \\
HHZ & 1147.8 & 1623.6 & 672.0 & -1.27 & 0.00 \\
IR36 & 1304.2 & 1737.3 & 871.0 & -1.00 & 0.00 \\
& & & & & \\
HHZ & 178.2 & 118.0 & 238.4 & 1.01 & 0.00 \\
IR36 & 184.9 & 164.3 & 205.5 & 0.32 & 0.05 \\
HHZ & 1872.9 & 2570.7 & 1175.2 & -1.13 & 0.00 \\
IR36 & 1251.4 & 1482.6 & 1020.2 & -0.54 & 0.00 \\
HHZ & 123.1 & 178.3 & 67.9 & -1.39 & 0.00 \\
IR36 & 202.3 & 267.6 & 136.9 & -0.97 & 0.00 \\
\hline & & & & &
\end{tabular}




\section{Table 5 (on next page)}

Table 5. Gene expression of DEGs in starch and sucrose metabolism in SHR 
2 Table 5. Gene expression of DEGs in starch and sucrose metabolism in SHR

\begin{tabular}{|c|c|c|c|c|c|c|c|}
\hline ID & Gene annotation & $\begin{array}{c}\text { Cultiva } \\
\mathrm{r}\end{array}$ & baseMean & IR36_32 & $\begin{array}{c}\text { IR36_4 } \\
0\end{array}$ & $\log 2$ FoldChange & $\begin{array}{c}\mathrm{P}- \\
\text { value }\end{array}$ \\
\hline BGIOSGA01057 & \multirow{2}{*}{ Sucrose synthase } & $\mathrm{HHZ}$ & 14318.1 & 18545.9 & 10090.4 & -0.88 & 0.00 \\
\hline 0 & & IR36 & 13352.6 & 18616.3 & 8088.8 & -1.20 & 0.00 \\
\hline BGIOSGA02614 & \multirow{2}{*}{ Sucrose synthase } & HHZ & 13.5 & 16.5 & 10.4 & -0.67 & 0.24 \\
\hline 0 & & IR36 & 16.8 & 24.7 & 8.9 & -1.47 & 0.01 \\
\hline BGIOSGA02697 & \multirow{2}{*}{ trehalose-6-phosphate synthase, putative, expressed } & HHZ & 651.9 & 572.0 & 731.7 & 0.36 & 0.12 \\
\hline 6 & & IR36 & 691.3 & 399.7 & 982.9 & 1.30 & 0.00 \\
\hline BGIOSGA00918 & \multirow{2}{*}{ trehalose-6-phosphate synthase, putative, expressed } & HHZ & 731.2 & 562.7 & 899.8 & 0.68 & 0.01 \\
\hline 1 & & IR36 & 988.8 & 578.3 & 1399.3 & 1.28 & 0.00 \\
\hline BGIOSGA03079 & \multirow{2}{*}{ trehalose-6-phosphate synthase, putative, expressed } & HHZ & 2.2 & 1.9 & 2.4 & 0.38 & 0.98 \\
\hline 6 & & IR36 & 4.3 & 0.0 & 8.6 & Inf & 0.00 \\
\hline BGIOSGA00050 & \multirow{2}{*}{ Trehalose-6-phosphate phosphatase } & HHZ & 175.0 & 229.9 & 120.2 & -0.94 & 0.00 \\
\hline 9 & & IR36 & 179.2 & 268.6 & 89.9 & -1.58 & 0.00 \\
\hline BGIOSGA03138 & \multirow{2}{*}{ beta-amylase, putative, expressed } & HHZ & 19.3 & 17.7 & 20.8 & 0.23 & 0.65 \\
\hline 5 & & IR36 & 28.0 & 17.4 & 38.5 & 1.15 & 0.01 \\
\hline
\end{tabular}

3 


\section{Figure 1}

Figure 1. Effects of high temperature on panicle development.

(a) young panicle morphologies after $9 \mathrm{~d}$ of high-temperature treatment; and (b) panicle morphologies at the heading stage after high-temperature treatment. Bars $=0.5 \mathrm{~cm}$ in (a) and $3 \mathrm{~cm}$ in (b). 


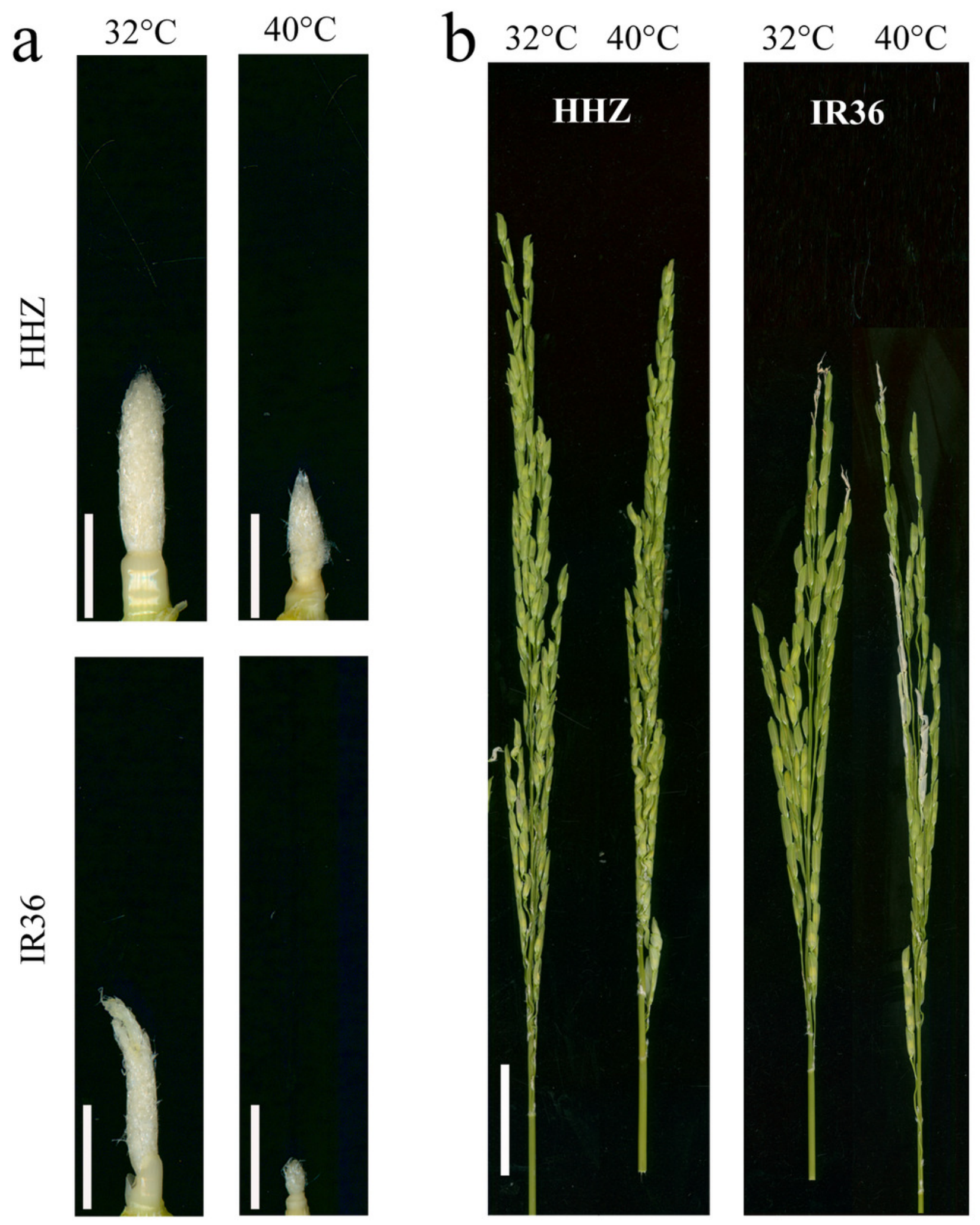


Figure 2

Figure 2. Gene expression in the four comparison groups.

(a) HHZ_32 vs HHZ_40, (b) IR36_32 vs IR36_40, (c) IR36_40 vs HHZ_40, and (d) IR36_32 vs HHZ_32. Red (upregulated) and blue (downregulated) dots indicate significant differences in gene expression, whereas gray dots represent genes with no significant difference in expression.

(a)

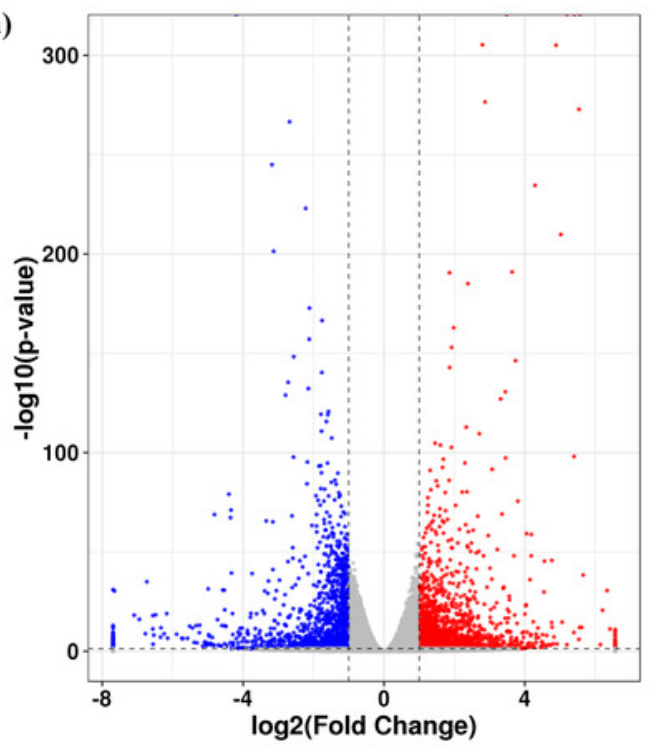

(c)

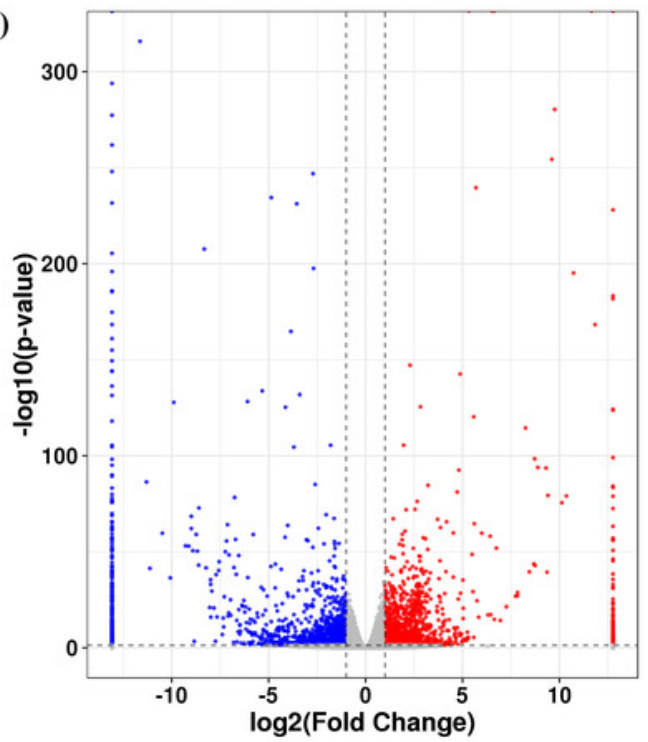

Control: HHZ_32 Case: $\mathrm{HHZ}_{4} 40$

Down (1548) NoDiff (24908) Up (1794)

Control: IR36_40 Case: $\mathrm{HHZ}_{-} 40$

Down (1541) NoDiff (25658) Up (1408)

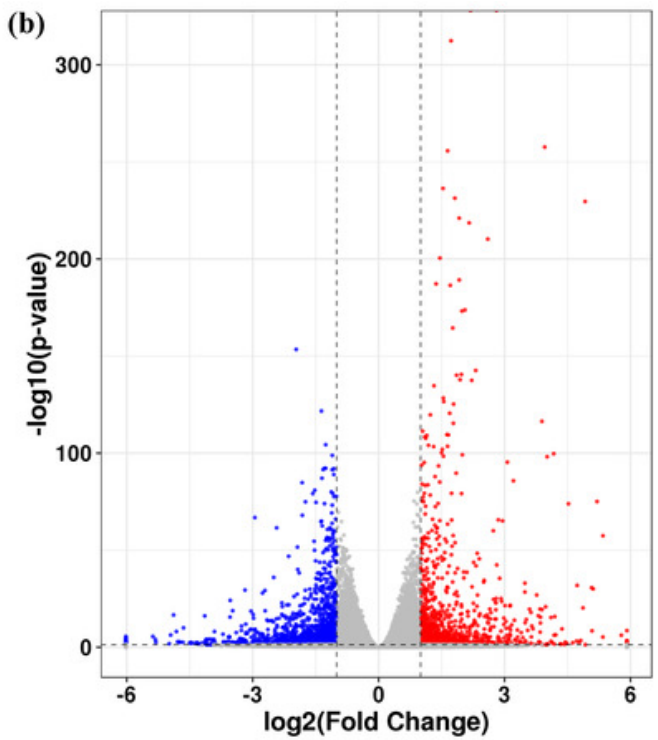

Control: IR36_32 Case: IR36_40

Down (1329) NoDiff (26610) Up (1140)

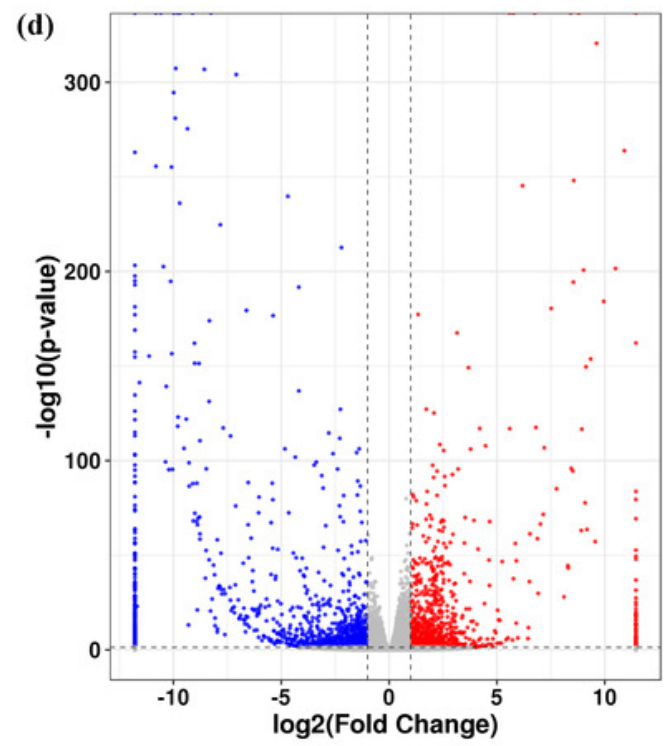

Control: IR36_32 Case: HHZ_32 Down (1571) NoDiff (26734) Up (893) 
Figure 3

Figure 3. Venn diagrams for DEGs in the four comparison groups.

(a) HHZ_32 vs HHZ_40, (b) IR36_32 vs IR36_40, (c) IR36_40 vs HHZ_40, and (d) IR36_32 vs HHZ_32. 2.

(b)

(c)

(a)

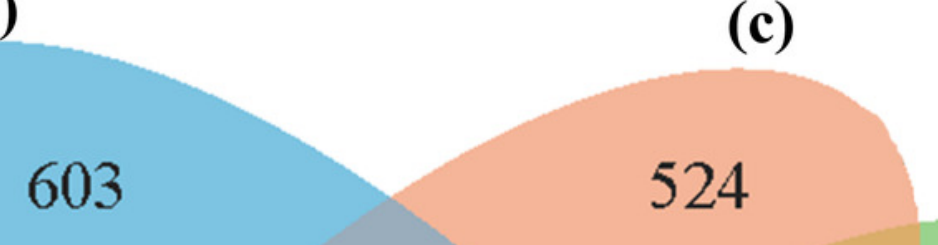

(d)

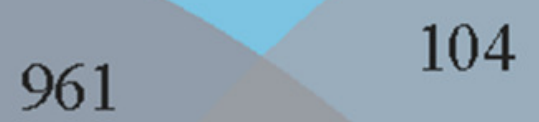

1167

1157

190

138

402

143

531

254

152

76

132 
Figure 4

Figure 4. Enriched GO terms $(P<0.05)$ of all DEGs.

(a) biological process, (b) cellular component, and (c) molecular function. 

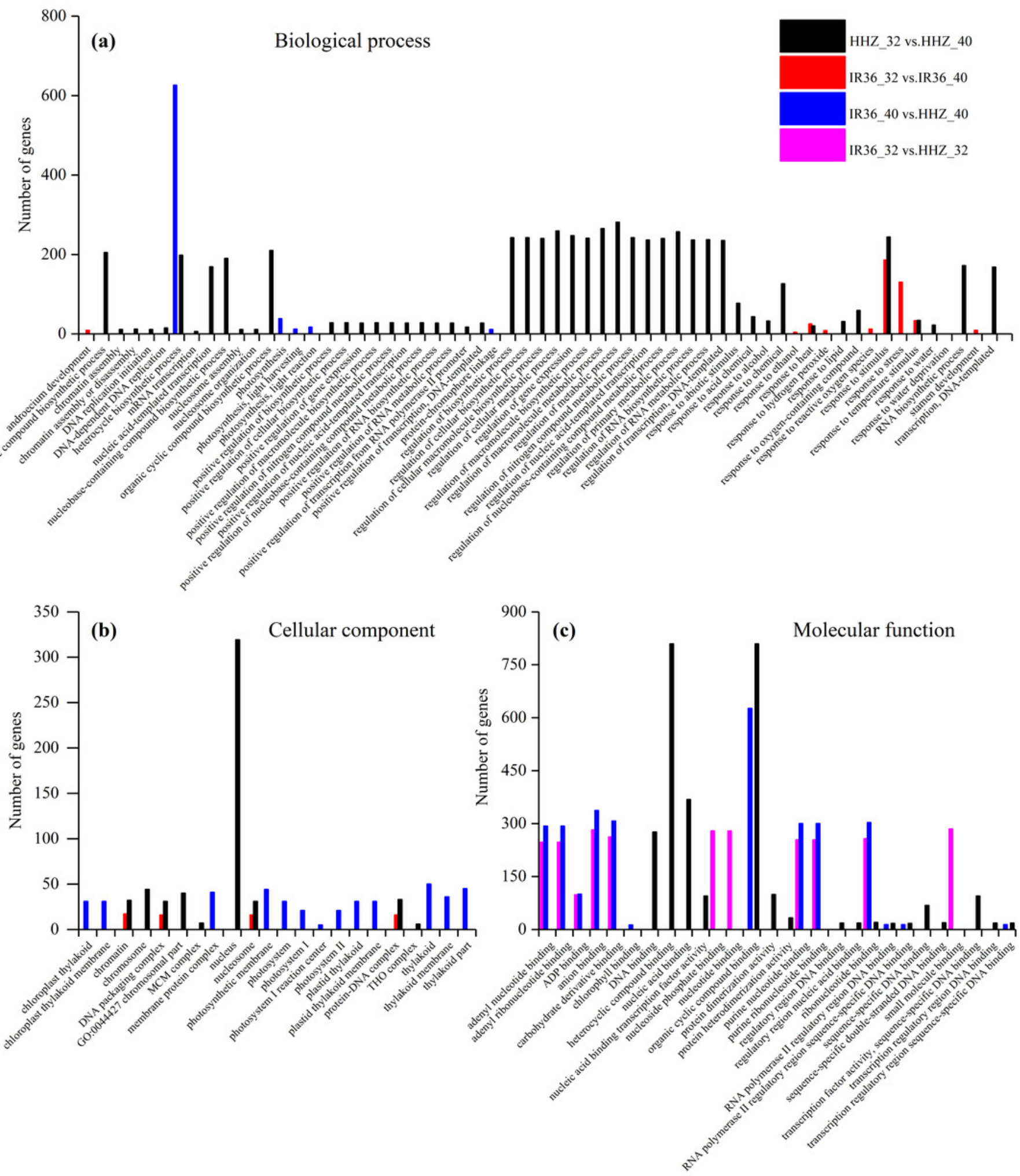
Figure 5

Figure 5. Enriched GO terms $(P<0.05)$ of DEGs in RHR and CHR.

(a) biological process, (b) cellular component, and (c) molecular function. 


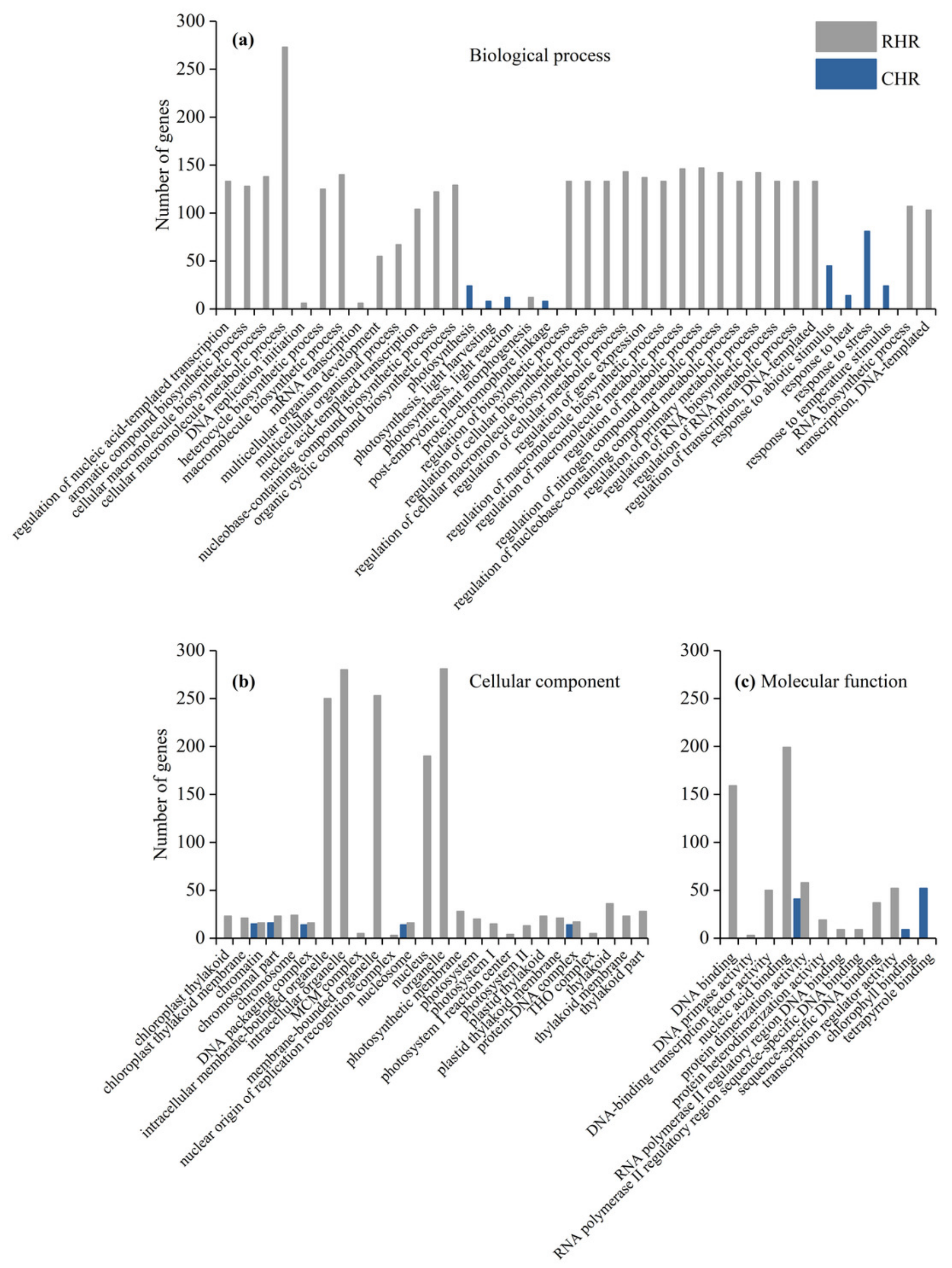


Figure 6

Figure 6. KEGG enrichment analysis of all DEGs.

(a) HHZ_32 vs HHZ_40, (b) IR36_32 vs IR36_40, (c) IR36_40 vs HHZ_40, and (d) IR36_32 vs HHZ_32.
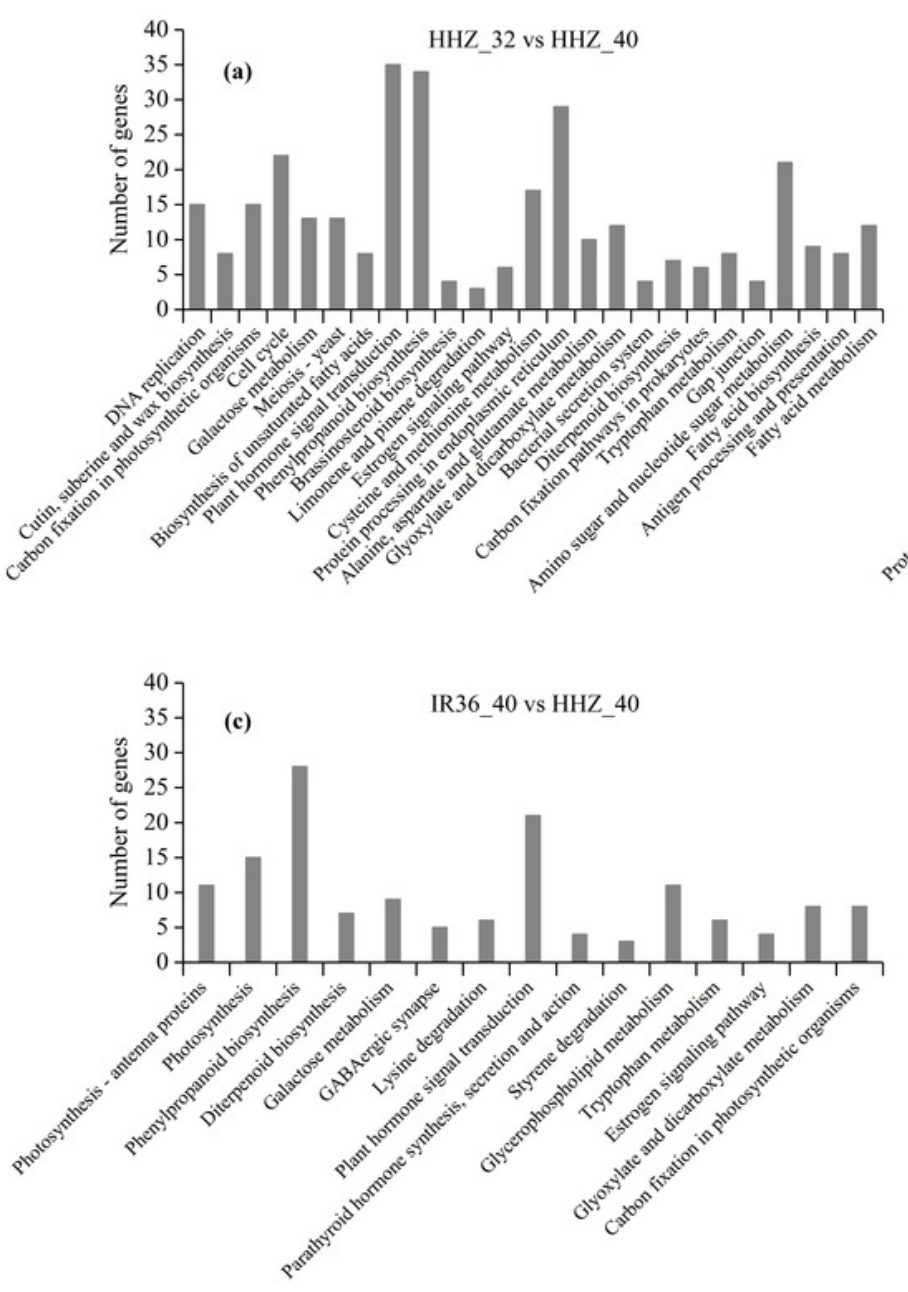
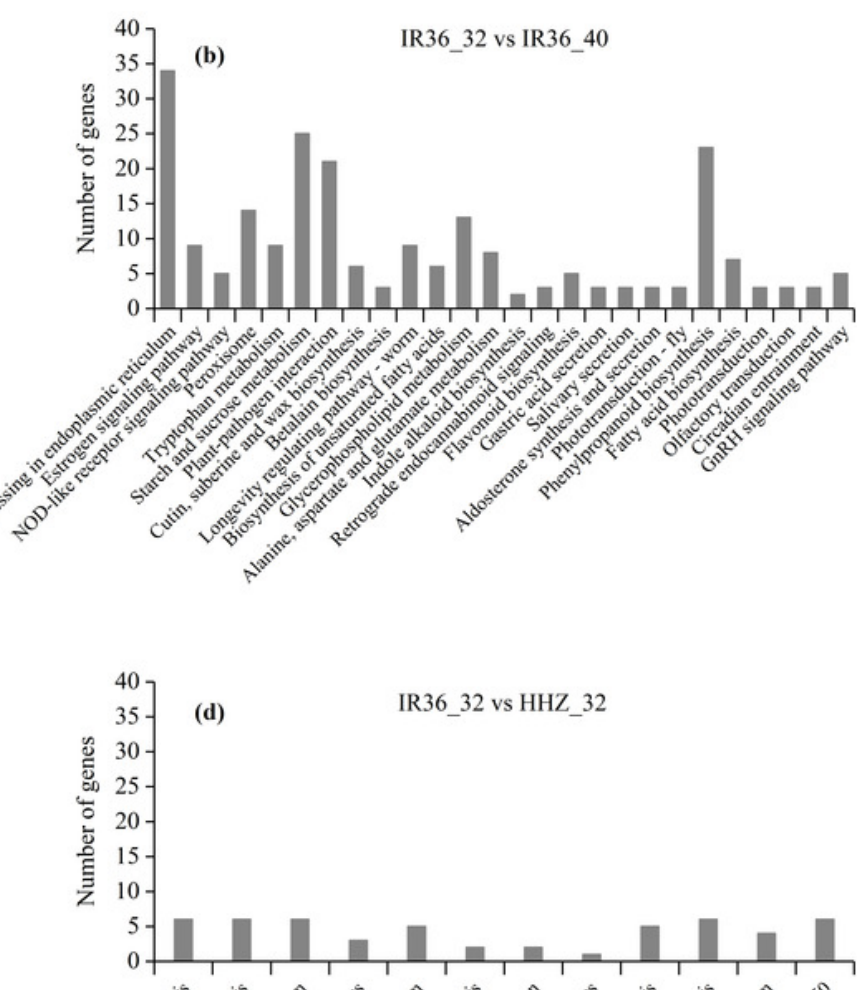

(d)

IR36_32 vs HHZ_32

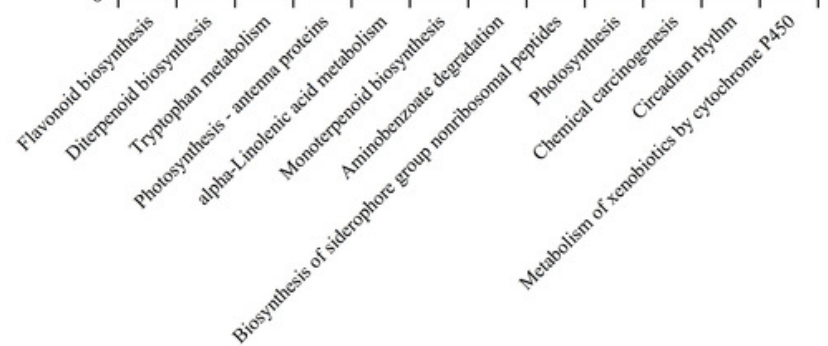


Figure 7

Figure 7. KEGG enrichment analysis for heat stress responsive genes from the three categories.

(a) RHR, (b) SHR, and (c) CHR.
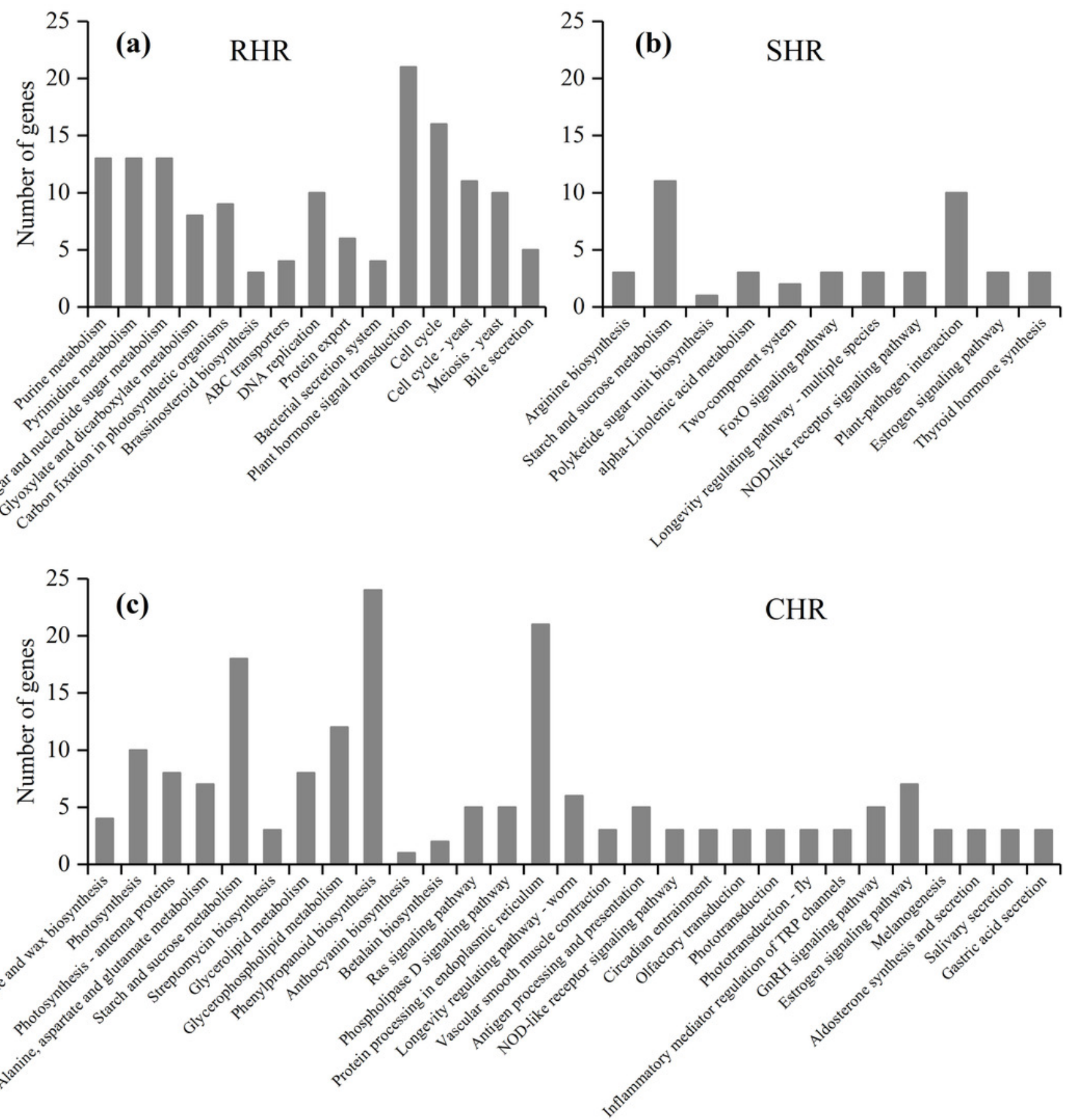
Figure 8

Figure 8. Gene expression levels determined by RNA-Seq and qRT-PCR.

(a) HHZ_32 vs HHZ_40, (b) IR36_32 vs IR36_40, (c) IR36_40 vs HHZ_40, and (d) IR36_32 vs HHZ_32. 

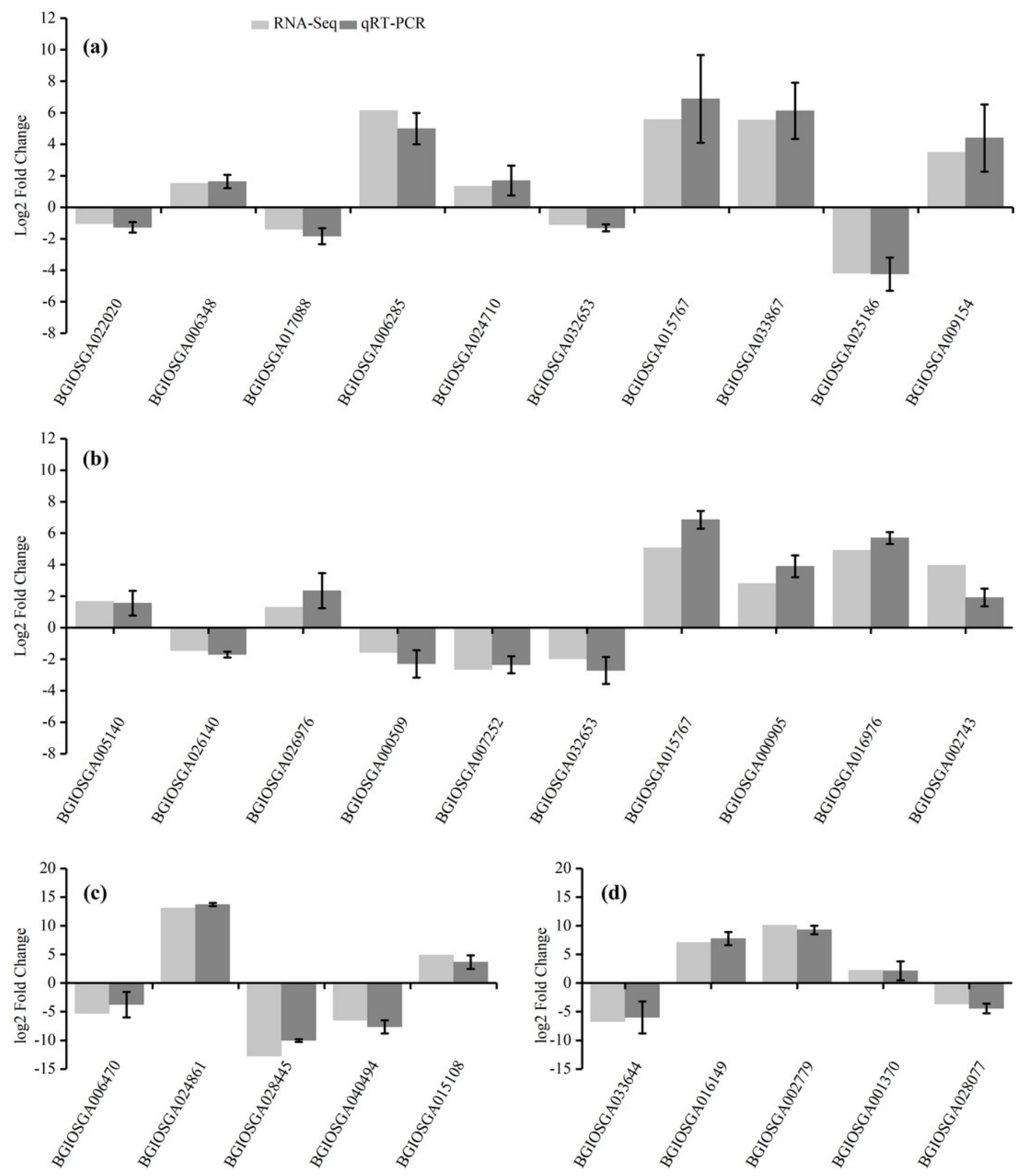